\title{
Diffusion du coton génétiquement modifié en Chine : leçons sur les facteurs et les limites d'un succès
}

Dissemination of GM cotton in China: lessons on the factors and limitations of its success

Michel Fok Ah Chuen, Weili Liang, Guiyan Wang et Yuhong Wu

\section{(2) OpenEdition}

Journals

Édition électronique

URL : http://journals.openedition.org/economierurale/3112

DOI : 10.4000/economierurale.3112

ISSN : 2105-2581

Éditeur

Société Française d'Économie Rurale (SFER)

Édition imprimée

Date de publication : 5 janvier 2005

Pagination : $5-32$

ISSN : 0013-0559

Référence électronique

Michel Fok Ah Chuen, Weili Liang, Guiyan Wang et Yuhong Wu, « Diffusion du coton génétiquement modifié en Chine : leçons sur les facteurs et les limites d'un succès», Économie rurale [En ligne], 285 | Janvier-février 2005, mis en ligne le 05 janvier 2007, consulté le 01 mai 2019. URL : http:// journals.openedition.org/economierurale/3112; DOI : 10.4000/economierurale.3112

Ce document a été généré automatiquement le 1 mai 2019.

(c) Tous droits réservés 


\section{Diffusion du coton génétiquement modifié en Chine : leçons sur les facteurs et les limites d'un succès}

Dissemination of GM cotton in China: lessons on the factors and limitations of its success

Michel Fok Ah Chuen, Weili Liang, Guiyan Wang et Yuhong Wu

\section{Introduction}

1 L'intérêt de l'utilisation de semences de variétés génétiquement modifiées, notamment dans les pays en développement (PVD), est objet de débat passionné entre partisans et adversaires. Le présent article vise à apporter une contribution au débat à partir des résultats d'une enquête récente sur la diffusion du coton génétiquement modifié (CGM) en Chine et à souligner que, en dépit de plusieurs aspects positifs, il convient de se garder d'une extrapolation abusive de l'expérience chinoise aux autres PVD. Le succès de la diffusion du CGM en Chine peut être perçu par la rapidité de son adoption par la quasitotalité des paysans dans les provinces où son utilisation est autorisée dans la vallée du fleuve Jaune. Ce succès procède de qualités intrinsèques du CGM en réponse au phénomène de résistance d'un ravageur important (Helicoverpa armigera) ${ }^{1}$ aux insecticides. Ces qualités sont certes importantes, mais nous pensons qu'elles n'ont $\mathrm{pu}$ s'exprimer qu'à la faveur de facteurs complémentaires, notamment d'ordre institutionnel. Pour éviter que les PVD ne prennent une décision relative à l'utilisation du CGM à partir d'une perception erronée de la valeur de l'expérience en Chine, il est important d'expliciter les facteurs institutionnels ayant influencé la réussite de la diffusion du CGM dans ce pays et d'analyser les chances de les voir répliquer dans d'autres PVD.

2 La production cotonnière revêt un caractère stratégique en Chine, premier producteur mondial: elle fournit la matière première à une industrie textile dont la puissance 
exportatrice est singulièrement mise à l'index depuis la fin des quotas autorisés par l'accord Multifibre. Cette production a profité de la réforme de la politique agricole mise en œuvre en Chine à partir de 1978, pour atteindre un record historique en 1984 qui a induit aussi la première crise du marché mondial de coton depuis la fin de la Seconde Guerre mondiale. La production devint alors plus fluctuante. Elle déclina à partir du début des années 1990 lorsque le ravageur principal, H. armigera, est devenu résistant aux insecticides. Cela fut particulièrement vrai dans la région où la production était traditionnellement dominante (vallée du fleuve Jaune), et ce jusqu'à la commercialisation du CGM, en 1998.

3 La première section de cet article fournit les éléments pour appréhender les caractéristiques de la production cotonnière en Chine, en insistant, en particulier, sur la protection phytosanitaire ainsi que sur les conditions d'utilisation des semences et de diffusion des variétés avant l'avènement du CGM.

4 L'intérêt et la pertinence de la diffusion des variétés génétiquement modifiées ont fait l'objet de nombreux travaux. Ces travaux se rapportent plus particulièrement aux pays développés (États-Unis, Australie), mais quelques études concernent des pays émergents (Argentine) ou des pays considérés comme des PVD (Chine, Afrique du Sud et, plus récemment, l'Inde). Nous consacrons la deuxième section à restituer la portée et les limites des résultats de ces travaux, en nous concentrant notamment sur le cas de l'utilisation du CGM résistant aux ravageurs.

5 Pour pallier l'insuffisance de restituer l'expérience chinoise dans le domaine du CGM par les seuls résultats de rentabilité comparée, la troisième section vise à situer l'utilisation du CGM par les paysans chinois dans leur environnement institutionnel, responsable de conditions d'utilisation beaucoup plus favorables que dans la plupart des pays. À cette fin, à partir des résultats de notre enquête menée au cours des campagnes 2002 et 2003, dans la province du Hebei (représentative des provinces cotonnières de la vallée du fleuve Jaune), nous confirmerons d'abord l'efficacité et la rentabilité de l'utilisation du CGM, bien que nous soyons dans une situation d'adoption généralisée qui ne facilite pas les comparaisons. Nous soulignerons ensuite les particularités de l'environnement institutionnel qui rendent cette adoption plus favorable pour les paysans.

Enfin, dans l'optique de cerner le degré d'extrapolation possible de l'expérience chinoise dans les autres PVD, la dernière section propose une catégorisation des facteurs favorables à l'utilisation du CGM par les paysans de ces pays. En mettant en évidence que peu de PVD, voire de pays émergents, pourraient les réunir à court terme ou moyen terme, nous évoquerons la pertinence d'une nouvelle forme de partenariat public-privé pour surmonter un tel écueil.

\section{Principales caractéristiques de la production cotonnière en Chine}

\section{Une production majeure}

7 Le coton provient de la culture du cotonnier, espèce arbustive de la famille des malvacées mais cultivée annuellement. À maturité, les fruits, appelés capsules, s'ouvrent pour exposer les graines qui sont récoltées manuellement ou mécaniquement. Les fibres de coton sont en fait portées par l'épiderme des graines, d'où le nom de coton-graine pour la 
partie récoltée. La séparation de la fibre de la graine ${ }^{2}$ est réalisée dans une opération appelée égrenage donnant un rendement de l'ordre de $40 \%$ de fibre à l'échelle mondiale, pour environ $55 \%$ de graines. L'égrenage, qui est aussi l'opération nécessaire pour disposer des semences, se fait au moyen de machines de capacités très variables. Plusieurs pays asiatiques, et c'est plus particulièrement le cas de la Chine, ont conçu et diffusé des machines de très faibles capacités, adaptées aux volumes de production d'un village ou d'une petite entreprise. Cette particularité a une incidence pour le sujet que nous abordons, dans la mesure où les capacités d'égrenage, ou de séparation de semences, sont très décentralisées et donc difficiles à contrôler.

La production de coton a une longue histoire en Chine. C'est dans ce pays que nous avons situé la première opération de développement de cette production avec l'implication de l'État au XVII ${ }^{e}$ siècle (Fok, 1997). La région traditionnelle de production se situe dans la vallée du fleuve Jaune. Elle était la principale région de production jusqu'au milieu des années 1980, avant d'être rattrapée par la province du Xinjiang, à l'ouest du pays, où la production n'a démarré qu'à partir des années 1970. La vallée du fleuve Yangse, plus au sud, est la troisième région de production où celle-ci tend à régresser : les températures excessives en été s'avèrent néfastes à la fructification du cotonnier alors que d'autres cultures peuvent être plus rentables.

Depuis une vingtaine d'années, la Chine est devenue le premier pays producteur, devançant les États-Unis. C'est aussi de loin le premier pays consommateur en raison du développement de l'industrie textile cotonnière. Sur le marché mondial, la Chine est importateur structurel et c'est sa demande en importation, très fluctuante, qui est un déterminant majeur du prix mondial (tableau 1). L'adoption du CGM en Chine, avec la réduction des importations qui peut résulter du gain de productivité associé à cette adoption, est ainsi considérée comme une menace pour les pays structurellement exportateurs.

Tableau 1. Production, consommation et importation de coton en Chine

\begin{tabular}{|c|c|c|c|c|c|c|}
\hline & Surface & Rendement & Equivalent & Production & Consommation & $\begin{array}{l}\text { Importation } \\
\text { (1 000 tonnes) }\end{array}$ \\
\hline & & fibre & coton-graine & fibre (tonne) & (1000 tonnes) & (1000 tonnes) \\
\hline & & (kg/ha) & (kg/ha) & & & \\
\hline 1990 & 5588 & $80 ?$ & 2306 & 4510 & 4225 & 480 \\
\hline 1991 & 6539 & 869 & 2481 & 5680 & 4250 & 355 \\
\hline 1992 & 6835 & 660 & 1886 & 4510 & 4600 & 53 \\
\hline 1993 & 4985 & 755 & 2156 & 3760 & 4600 & 176 \\
\hline 1994 & 5528 & 770 & 2199 & 4250 & 4500 & 884 \\
\hline 1995 & 5421 & 428 & 1221 & 4760 & 4400 & 663 \\
\hline 1996 & 4722 & 890 & 2541 & 4203 & 4600 & 787 \\
\hline 1997 & 4491 & 1025 & 2927 & 4603 & 4500 & 402 \\
\hline 1998 & 4459 & 1010 & 2884 & 4501 & 4400 & 78 \\
\hline 1999 & 3748 & 1022 & 2919 & 3828 & 4800 & 30 \\
\hline 2000 & 3600 & 1227 & 3506 & 4417 & 5050 & 52 \\
\hline 2001 & 4809 & 1108 & 3167 & 5324 & 5500 & 102 \\
\hline 2002 & 4180 & 1177 & 3363 & 4920 & 5600 & 400 \\
\hline
\end{tabular}

Sources: International Cotton Advisory Committee (ICAC) et China Agriculture Yearbooks. 


\section{Une production intensive}

10 La production cotonnière est issue quasi exclusivement du petit paysannat cultivant en moyenne 0,8 ha. Dans la vallée du fleuve Jaune, le coton occupe environ $40 \%$ de la surface cultivée, soit un tiers d'hectare (Liang et al., 2004). La production très intensive est responsable d'un rendement élevé, de l'ordre de $3500 \mathrm{~kg} / \mathrm{ha}$ de coton-graine. La Chine se place ainsi parmi les trois premiers pays aux meilleurs rendements, aux côtés de l'Australie (pays exportateur) et de l'Israël (pays à production marginale). En considérant les pays à agriculture de petit paysannat, la Chine est assurément de loin le pays le plus performant en terme de productivité de la terre.

11 La production cotonnière bénéficie au moins d'une irrigation d'appoint, dans toutes les régions de production en Chine. Dans l'ouest du pays, dans une région semi-aride, l'irrigation est totale. Les techniques culturales sont assez différenciées suivant les trois grandes régions de production. La culture est installée par semis dans les deux principales régions de production, mais dans la vallée du Yangse, elle l'est surtout par une technique de transplantation largement diffusée à la fin des années 1980. Partout, le coton est produit avec fort recours aux intrants chimiques. Hormis les herbicides dont l'usage paraît peu justifié par l'exiguïté des parcelles que les paysans parviennent à entretenir comme des lopins de jardin, les producteurs chinois utilisent engrais et insecticides en grandes quantités. Les doses d'engrais utilisées sont en moyenne de 530 unités fertilisantes ${ }^{3}$, avec fréquemment un surdosage en azote, voire également en phosphore, mais un sous-dosage en potassium. Le nombre de traitements contre les ravageurs varie dans le temps et dans l'espace. Dans la province du Xinjiang, à l'ouest du pays, les traitements insecticides n'étaient pas jugés nécessaires jusqu'au milieu des années 1990, mais les attaques de H. armigera s'y sont manifestées depuis, parfois de manière sévère (Sheng, 2000). Dans la vallée du fleuve Jaune que nous avons étudiée, avant l'avènement du CGM et après l'émergence de la résistance de $H$. armigera , il était courant de recenser 20 à 30 traitements contre ce ravageur, certains paysans déclarant avoir eu à traiter tous les deux jours. Le cotonnier dans cette région est aussi soumis à une infestation endémique de plusieurs maladies, dont les deux plus importantes sont la fusariose et la verticiliose. Les températures élevées et les doses élevées d'engrais conduisent à une forte croissance que les paysans chinois contrôlent depuis près de vingt ans par l'emploi de régulateur de croissance, en moyenne à trois reprises. En général, les différents produits insecticides, fongicides et régulateurs de croissance peuvent être mélangés à l'épandage en Chine. C'est une particularité qui ne rend pas aisée l'identification du temps de travail associé spécifiquement aux traitements insecticides et qui peut conduire à mésestimer l'économie en temps résultant de l'emploi du CGM.

12 Comme le montre le tableau 2, en valeur monétaire, le recours aux intrants en Chine est comparable à celui observé aux États-Unis (en dépit de coûts unitaires moindres). 
Tableau 2. Comparaison des coûts d'intensification en Chine et ailleurs ( $\$$ US/ha)

\begin{tabular}{lcrrrrrr}
\hline Pays et années & Chine (Province du Hebei & \multicolumn{1}{c}{ USA $^{*}$} & Mali & Bénin & Mozambique $^{*}$ \\
\hline & 2002 & 2003 & 1997 & 2001 & $\mathbf{1 9 9 8}$ & 2001 & 2000 \\
\hline Coût des produits chimique & 143 & 147 & 210 & 299 & 83 & 95 & 12 \\
Coût des semences & 42 & 51 & 36 & 48 & 0 & 0 & 0 \\
\hline Coût des autres intrants & 69 & 69 & 23 & & 0 & 0 & 0 \\
\hline Coût total des intrants physiques & 254 & 267 & 269 & 347 & 83 & 95 & 12 \\
\hline
\end{tabular}

Coûts établis pour les fermes à niveau moyen de coût en 1997, et pour la région du Mississipi Portal en 2001, non inclus les frais de redevance d'emploi des semences. ** Coûts établis pour la Province du Cabo Delgado Province, au Nord du Mozambique.

Sources: Brooks (2001), Fok et al. (2004), ICAC (2001).

\section{Une intensification longtemps soutenue}

13 Le niveau d'intensification rencontrée en Chine résulte du soutien que la production cotonnière a bénéficié, en particulier depuis la réforme de la politique agricole de 1978. Ce soutien repose sur la subvention à l'usage des intrants et à la garantie d'un prix attractif. Ce soutien exprime certainement la volonté d'augmenter la production pour satisfaire les besoins importants de l'industrie textile cotonnière génératrice de devises dont la Chine avait grand besoin à cette époque ${ }^{4}$, ce qui est différent de l'idée d'une production cotonnière taxée au profit de l'industrie textile (Nyberg et al., 1994).

Le soutien à l'utilisation des intrants chimiques, plus particulièrement à celle des engrais, prenait la forme de diverses subventions octroyées aux niveaux national et provincial, voire même d'échelons administratifs inférieurs. Le gasoil pour le fonctionnement des tracteurs était généralement subventionné localement. Ce soutien a commencé à se réduire à partir de la deuxième moitié des années 1990 et a totalement disparu ${ }^{5}$ depuis l'entrée de la Chine à l'Organisation mondiale du commerce (OMC), condition revendiquée par le lobby cotonnier des États-Unis.

La production cotonnière est l'une des productions agricoles pour lesquelles la fixation administrative du prix d'achat aux producteurs est restée le plus longtemps en vigueur. La fixation du prix du coton a toujours été déterminée en considérant l'écart de productivité avec les principales céréales (blé et maiis) pour lui assurer une attractivité suffisante. La libéralisation du marché des céréales est cependant intervenue dès le milieu des années 1990, en induisant une fluctuation importante des prix. Le coton a bénéficié ainsi d'une garantie de prix qui a renforcé son intérêt pour les producteurs jusqu'en $2000 / 2001$. Depuis cette date, il n'y a plus de soutien direct au prix d'achat du coton sous la forme d'une garantie de prix : le prix d'achat payé aux paysans fluctue au jour le jour en fonction de la demande de l'industrie textile nationale. Néanmoins, du fait d'un certain contrôle de l'importation du coton que la Chine a réussi à préserver, même après son entrée à l'OMC, on peut dire qu'il reste un soutien indirect au prix d'achat.

\section{Progression de la production sapée par la résistance d'un ravageur}

Le coton est une culture soumise de tout temps aux attaques de nombreux ravageurs, ce qui le faisait percevoir comme une "culture impossible» (Michotte, 1924). On distingue principalement deux groupes de ravageurs du cotonnier, les chenilles de la capsule et les insectes piqueurs-suceurs. Les attaques du premier groupe, incluant $H$. armigera, sont souvent considérées comme ayant le plus d'incidence sur le rendement. La mise en œuvre d'insecticides chimiques à la fin des années 1950 a été à l'origine du progrès de 
rendement dans le monde : il s'agissait de la première génération d'insecticides à base d'organochlorés très rémanents et à toxicité très élevée. On a évolué ensuite vers l'utilisation de produits insecticides à action plus ciblée sur des ravageurs particuliers et à toxicité moindre. En Chine, la réforme de la politique agricole a coïncidé avec le lancement d'une nouvelle famille de produits insecticides à base de pyréthrinoïdes de synthèse pour contrôler les infestations de chenilles. L'utilisation de ces insecticides, sans la moindre coordination en Chine (puisque la réforme agricole visait d'abord à flatter les initiatives individuelles après tant d'années d'inefficacité collective), a provoqué une forte résistance du principal ravageur, $H$. armigera, en moins de dix années d'utilisation dans la vallée du fleuve Jaune. Si l'émergence de cette résistance n'était pas forcément plus rapide que celle observée en Thaïlande ou en Australie, elle était plus violente par la chute de rendement induite en dépit de l'accroissement du nombre de traitements insecticides réalisés.

Ce phénomène de résistance est arrivé à son paroxysme en 1993 en provoquant une forte baisse de rendement. Les années qui ont suivi n'ont pas engendré les mêmes chutes de rendement, mais les paysans ont dû augmenter le nombre de traitements insecticides pour une rentabilité moindre. Cette situation est responsable du retrait de la production cotonnière dans la province du Hebei, représentative de la région cotonnière de la vallée $\mathrm{du}$ fleuve Jaune (figure 1). Elle explique une attente pour une solution technique, notamment avec des variétés résistantes. La demande pour du CGM était ainsi latente.

\section{Forte présence de l'État dans la création des variétés et la diffusion des semences}

Le soutien à l'intensification se retrouve aussi dans la forte présence de l'État dans la création variétale, avec des effets positifs pour la constitution d'un riche germplasme mais des effets plus discutables dans le domaine des semences.

Comme pour toutes les espèces de grandes cultures en Chine, la création variétale est le fait d'un grand nombre d'instituts de recherche opérant à l'échelle nationale, provinciale ou à des échelons administratifs inférieurs (districts et comtés). L'institut de recherche cotonnière, relevant de l'Académie nationale des sciences agricoles, opère à l'échelon national tout en étant localisé dans la province du Henan. Dans cette province, comme dans toutes les autres provinces cotonnières, les académies provinciales des sciences agricoles comprennent un institut spécialisé sur le coton (ou sur les cultures industrielles). À ces instituts s'ajoutent les stations de recherche directement rattachées à l'administration des districts. L'étendue des travaux de recherche est bien entendu variable entre les divers types d'institut, mais ils intègrent tous un programme de création variétale à l'origine d'un grand nombre de variétés. Jusqu'à la commercialisation du CGM, toutes ces institutions de recherche fonctionnaient en partenariat se traduisant notamment par un grand échange de matériel végétal. Cette grande décentralisation de la création variétale est à l'origine d'un grand nombre de variétés proposées aux paysans chinois ${ }^{6}$, reposant sur les qualités de productivité et de résistance aux maladies.

Un fait marquant concerne l'introgression de gènes (en particulier Bt) que les petits instituts locaux ont pu bénéficier grâce à la coopération des centres provinciaux: l' Institute of Industrial Crops (l'Académie provinciale des sciences agricoles du Jiangsu) a certainement introgressé le gène Bt dans beaucoup de variétés d'instituts locaux, sans forcément se limiter à la construction génique d'une équipe chinoise (cf. infra). On peut 
penser que cette pratique de coopération ou de complaisance soit révolue pour se conformer à la loi ${ }^{7}$, il n'empêche que de petits instituts locaux ont pu obtenir leurs premières variétés de coton Bt sans avoir eu à payer de royalties. Ce ne serait plus le cas aujourd'hui, le paiement de royalties est requis en respect de la propriété intellectuelle $\mathrm{du}$ gène $\mathrm{Bt}$ et des engagements de la Chine vis-à-vis de l'OMC, mais nous n'avons pas la possibilité de vérifier le taux évoqué de $10 \%$ du prix des semences.

21 En Chine, l'impact positif de la création variétale a été accentué par la mise au point de nouvelles techniques culturales. En même temps que l'engagement des travaux en biotechnologies, au milieu des années 1980, les instituts chinois ont investi la création de variétés hybrides dont les premières variétés furent finalisées au début des années 1990, soit au même moment que l'émergence de la résistance de H. armigera aux insecticides.

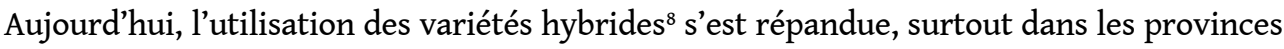
de la vallée du fleuve Yangse, à la faveur de la technique de transplantation qui permet de réduire fortement les doses de semences pour en diminuer les coûts. Il faut donc retenir qu'en raison du dynamisme de la recherche cotonnière en Chine, les paysans ont bénéficié d'un foisonnement de nouveautés techniques (on peut mentionner aussi le mulching plastique, la régulation de la croissance du cotonnier...) qui ont contribué à renforcer l'expression des progrès génétiques. Cette situation indique qu'il est difficile d'expliquer l'augmentation observée du rendement par la seule technologie du CGM.

En dépit de la qualité indéniable des variétés, les semences n'étaient pas valorisées de manière correcte avant la commercialisation du CGM. À notre connaissance, le sujet de la commercialisation des semences était peu étudié avant l'avènement du CGM. Nos entretiens mettent en évidence qu'il n'y avait pas de marché organisé des semences. L'organisation de la distribution des semences était réalisée par le même service d'État qui se chargeait de l'achat des productions des paysans, manifestement avec une logique de subvention. Les semences étaient distribuées au niveau des centres d'égrenage, à un prix modique $(0,25-0,35 \$ / \mathrm{kg})$, sous une forme peu différenciée des graines tout venant. Les semences ne semblaient pas avoir de valeur spécifique et les paysans avaient pris une certaine habitude ${ }^{9}$ d'utiliser les graines de leur production qu'ils avaient conservées car ils étaient méfiants sur la qualité de celles qu'on leur distribuait dans les centres d'égrenage. Indéniablement, l'utilisation du CGM a fait profiter aux paysans des avantages de semences de qualité, c'est un acquis que nous pensons devoir être mis au crédit de Monsanto (Fok et al., 2005).

Peu de temps avant la commercialisation du CGM, le cadre institutionnel relatif à la propriété intellectuelle des variétés et à la diffusion des semences a connu un changement notable. Les principes du règlement national portant sur les semences et la propriété intellectuelle sur les variétés ont été énoncés en décembre 1989, suivis par la publication d'une sorte de décret d'application en juin 1991. La loi nationale sur les semences végétales est promulguée en fin 2000. La commercialisation du CGM est intervenue entre-temps, en 1998. Il serait trop fastidieux de procéder à l'analyse du cadre institutionnel relatif aux semences en relation avec les trois décisions du gouvernement central mais retenons que ce cadre a été défini dès le début des années 1990 et qu'il devait apparaitre suffisamment crédible pour inciter Monsanto à investir quelques années plus tard. 


\section{Des acquis importants dans le domaine des biotechnologies}

La solution du CGM pour surmonter le problème de la résistance du principal ravageur du cotonnier peut paraître naturelle en raison des acquis de la Chine dans le domaine des biotechnologies.

La Chine a été le premier pays à utiliser à grande échelle des variétés génétiquement modifiées, d'abord pour la culture du tabac (de 1992 à 1995). La recherche en biotechnologie y est fort développée. Durant la période 1996-2000, le Chinese Office of Genetic Engineering Safety and Administration a autorisé l'expérimentation ou la commercialisation de 251 OGM dans les domaines végétal, animal et de micro-organismes recombinés (Teng et al., 2004). Ce sont les résultats des travaux qui ont connu une accélération au début des années 1980, avec le lancement du Programme «863", poursuivi ensuite par le Programme « 973 », et qui ont permis aux équipes de recherche chinoises d'identifier de nombreux gènes, de bâtir de nouvelles constructions géniques qui leur sont propres et de maîtriser une méthode de transformation génétique par l'introgression au niveau du tube pollinique peu après la fécondation.

De manière générale, ces programmes ont permis aux Chinois d'explorer les promesses biotechnologiques sur un grand nombre de productions agricoles. La Chine se distingue ainsi par des travaux qui ne sont pas concentrés sur un nombre limité de cultures. Aujourd'hui, les acquis en biotechnologie concernent environ dix-huit espèces végétales relevant de plantes industrielles, de plantes vivrières, maraîchères ou florales (Huang et al ., 2003b) bien que l'utilisation ne soit à ce jour autorisée que sur quatre d'entre elles (coton, tomate, poivron et pétunia).

Dans le domaine du coton, l'équipe du professeur Guo San Dui de l'Académie des Sciences est propriétaire reconnue d'une construction Bt nouvelle, fondée sur la combinaison de séquence contrôlant la synthèse des toxines Cry 1B et Cry 1C. Les discussions avec certains chercheurs laissent penser que c'est ce gène qui est introduit dans les variétés de CGM de fabrication chinoise, à partir de l'exploitation poussée de la technique de transformation par l'introgression au niveau du tube pollinique. Nous n'avons pas connaissance de plainte de Monsanto contre l'usage non autorisé de ses gènes. Monsanto, une variété (SGK 321) avec deux gènes de résistance aux chenilles des capsules du cotonnier, en associant un gène Bt et un gène inhibiteur de protéase. L'efficacité et la durabilité de cette double résistance sont potentiellement meilleures que celles proposées par Monsanto à partir de l'association de deux gènes Bt.

Pour résumer, il convient de retenir que la production cotonnière est économiquement importante en Chine. C'est le fruit d'une agriculture de petit paysannat mais avec un haut niveau de rendement résultant d'une intensification poussée que l'État avait soutenue pendant très longtemps. En dehors du soutien direct aux producteurs à travers les subventions aux intrants et la garantie d'un prix au-dessus du prix mondial, l'appui de l'État s'est exprimé aussi par un système étoffé et décentralisé de création variétale responsable d'une richesse enviable de germplasme. En dépit d'une production performante, la Chine est structurellement importatrice de coton. La progression de la production a été perturbée depuis 1993 par le phénomène de résistance de H. armigera aux insecticides. Au niveau des paysans, ce phénomène a provoqué le retrait de la production cotonnière. À l'échelle du pays, il accroît les besoins en importation. La demande sociale 
pour une solution technique était alors forte. Les acquis techniques dans le domaine des biotechnologies et l'avancée institutionnelle en matière de reconnaissance de la propriété intellectuelle faisaient naturellement reposer l'espoir sur le CGM.

\section{Aperçu sur la rentabilité améliorée du CGM dans le monde}

\section{Caractéristiques et diffusion du CGM}

La commercialisation du CGM aux États-Unis, à partir de la campagne agricole 1996/97, précédant de peu la commercialisation en Australie puis en Afrique du Sud et en Chine, a généré un grand nombre de travaux pour cerner son intérêt économique. On peut dire que les travaux concernent tous les pays qui ont autorisé la commercialisation du CGM, même si les études du cas des États-Unis prédominent.

31 En 2003, le coton représentait $11 \%$ des surfaces en OGM dans le monde, et $23 \%$ de la surface en cette culture, avec une montée en puissance de la part de la Chine et de l'Inde (tableau 3). La commercialisation du CGM concerne deux types de caractère qui peuvent éventuellement être combinés pour donner les variétés de coton Bt résistant à un type de ravageur et de coton résistant à un herbicide spécifique (notamment le Round-Up Ready). L'autorisation à la commercialisation du CGM ne couvre pas forcément les deux types de variétés. En Afrique du Sud par exemple, il n'y a pas encore d'autorisation pour la diffusion des variétés résistantes aux herbicides. En Chine, il n'y a pas de commercialisation de ce type de variétés dont on peut discuter de l'intérêt en raison de l'exiguïté des parcelles. Ailleurs, en particulier aux États-Unis, la large diffusion du coton résistant aux herbicides est associée à l'intérêt ressenti pour une nouvelle méthode de culture du cotonnier, en rangs très serrés (Ultra Narrow Cotton). Pour ces raisons, nous nous concentrons ici sur les études de rentabilité d'utilisation du CGM résistant aux ravageurs (coton-Bt), auquel est associé l'intérêt d'une réduction des coûts du contrôle des chenilles de la capsule pour gagner en rentabilité.

Tableau 3. Evolution des surfaces en CGM dans le monde (en millions d'ha)

\begin{tabular}{|c|r|r|r|r|r|r|r|r|r|}
\hline & 1996 & 1997 & 1998 & 1999 & 2000 & 2001 & 2002 & 2003 & 2004 \\
\hline USA & 0,80 & 1,40 & 2,30 & 3,20 & 4,60 & 5,00 & 4,50 & 4,20 & 4,30 \\
\hline Australie & & & 0,08 & 0,12 & 0,12 & 0,14 & 0,07 & 0,09 & 0,25 \\
\hline Chine & & & 0,06 & 0,30 & 0,50 & 1,50 & 2,10 & 2,80 & 3,70 \\
\hline Mexique & & & 0,04 & 0,02 & 0,02 & 0,02 & 0,02 & 0,03 & \\
\hline Argentine & & & & 0,01 & 0,02 & 0,02 & 0,02 & 0,02 & \\
\hline Inde & & & & & & & 0,04 & 0,10 & 0,05 \\
\hline Autres & & & & 0,01 & 0,03 & 0,06 & 0,05 & 0,03 & \\
\hline
\end{tabular}




\begin{tabular}{|l|c|c|c|c|c|c|c|c|c|}
\hline Total & 0,80 & 1,40 & 2,48 & 3,66 & 5,29 & 6,74 & 6,80 & 7,27 & 9,00 \\
\hline $\begin{array}{l}\text { Surface } \\
\text { monde }\end{array}$ & 2 & 4 & 8 & 11 & 17 & 20 & 23 & 23 & 25 \\
\hline
\end{tabular}

Sources: International Service for the Acquisition of Agri-biotech Applications (ISAAA et ICAC). capsule) et non contre tous les ravageurs. La synthèse de la toxine Bt par les plantes de coton-Bt est surtout effective jusqu'à un certain stade du cotonnier, de sorte que l'efficacité du coton-Bt est en général manifeste contre les premières générations des ravageurs cibles. Cette synthèse est par ailleurs dépendante des conditions climatiques. Globalement, par l'utilisation du coton-Bt, on ne peut tabler sur une suppression totale des traitements insecticides contre les ravageurs cibles. Il convient de noter aussi qu'il y a plusieurs espèces de chenilles de la capsule. Les chenilles qui attaquent à partir de l'extérieur de la capsule (exocarpiques) sont les plus fréquentes mais celles qui attaquent à partir de l'intérieur sont les plus difficiles à détecter et donc à maitriser correctement par les traitements insecticides. Pour les chenilles exocarpiques, c'est l'espèce Heliothis zea qui prédomine aux États-Unis et contre laquelle le coton-Bt a été conçu, le degré d'infestation de ce ravageur est cependant variable entre les États cotonniers de ce pays.

Dans les autres pays où ce coton est utilisé, c'est l'espèce $H$. armigera également exocarpique, qui constitue le ravageur principal. Au lancement du coton-Bt, son efficacité dans tous les pays ne pouvait donc être considérée comme acquise partout.

\section{Trois groupes de travaux}

L'ensemble des travaux sur le CGM couvre principalement les États-Unis, l'Australie, l'Afrique du Sud, la Chine, l'Argentine, le Mexique et plus récemment l'Inde. Les études sur la Chine et l'Afrique du Sud, et bien sûr l'Inde, sont présentées fréquemment comme des études de cas de diffusion du CGM dans des PVD, et dont les résultats ont vocation d'éclairer le choix de cette catégorie de pays. L'ensemble des travaux peut être réparti en trois groupes complémentaires quoique déséquilibrés.

- Le premier groupe, le plus massivement représenté, concerne les études de rentabilité comparée entre le coton-Bt et le coton conventionnel, à partir de la méthode de budget partiel, dans le but de vérifier si le surcoût lié à l'utilisation de semences de coton-Bt est plus que compensé par la réduction des coûts du contrôle des ravageurs du cotonnier. Ces travaux (Bacheler et al., 1998 ; Carlson et al., 1998 ; Edens et al., 1998 ; Elena, 2001 ; Kerby, 2001, Marra et al., 2003) appréhendent la rentabilité à l'échelle de la parcelle et sont souvent réalisés à partir des dispositifs expérimentaux des universités agricoles aux États-Unis, parfois en association avec la firme Monsanto. Ils s'appuient parfois sur des enquêtes au téléphone auprès des fermiers qui doivent cultiver les deux types de coton, en conformité avec la démarche de prévention de la résistance aux toxines Bt. Ailleurs, les travaux sont fondés sur des enquêtes de terrain montées spécifiquement. C'est le cas de la Chine dans la vallée du fleuve Jaune, mais aussi en Argentine. On pourrait en dire de même pour les travaux rapportés en Afrique du Sud même si les données ont été collectées essentiellement à la commercialisation auprès d'une société cotonnière. Tous 
ces travaux ont concerné des pays où les semences étaient déjà commercialisées au prix du marché avant l'avènement du CGM, sans subvention, à l'exception notable de la Chine. - Le deuxième groupe de travaux concerne les enquêtes pour appréhender les perceptions des utilisateurs du coton-Bt. Ces enquêtes ont été spécifiquement réalisées aux États-Unis, en Inde et en Afrique du Sud (Bennett et al., 2004 ; Edens et al., 1998 ; Kirsten et al., 2002 ; Orphal, 2005 ; Qaim et al., 2003). Dans le cas de l'Afrique du Sud, les enquêtes ont concerné à la fois les grandes fermes commerciales (tenues par les fermiers blancs) et les petites exploitations familiales (tenues par les paysans noirs dans la province du Kwazulu Natal). En revanche, en dépit de la mise en place d'un dispositif important dans plusieurs provinces de la vallée du fleuve Jaune en Chine, les travaux rapportés n'ont pas visé à restituer les appréciations des paysans (Huang et al., 2002). Toutes ces enquêtes correspondent à la manière d'appréhender les impacts de l'utilisation du coton-Bt en se positionnant dans la perspective globale des utilisateurs, dans leurs contraintes techniques et non techniques à produire le coton. Elles correspondent à une « dimension exploitation » et ont permis de révéler des impacts qui n'étaient pas forcément anticipés au départ.

- Le troisième groupe de travaux correspond à une optique filière pour cerner la répartition des gains issus de l'utilisation. Ces travaux ont concerné essentiellement les États-Unis, le Mexique, la Chine et l'Afrique du Sud (Falck-Zepeda et al., 1999, 2000 ; Gouse et al., 2004 ; Taxler et al., 1999 ; Taxler et al., 2004) en prenant explicitement en compte les conditions d'utilisation du CGM, notamment l'obligation ou pas du versement d'une redevance d'emploi. La réalisation de ces travaux fait appel à des modèles mais elle aurait été possible, voire plus explicite, suivant la "méthode des effets » (Benhamou et al., 1992; Chervel et al., 1997 ; Fabre, 1997) ou la Matrice de comptabilité sociale.

Dans la réalité, même si beaucoup de travaux correspondent exclusivement au premier groupe, d'autres travaux sont à cheval sur deux des trois groupes que nous avons identifiés. Tous les travaux disposent d'une référence en coton conventionnel, soit parce que le cadre institutionnel d'utilisation du CGM dans certains pays permettait le maintien de ce coton (cas des États-Unis et de l'Australie notamment) soit parce que les études étaient réalisées en début de commercialisation du CGM avant la généralisation de son utilisation (Chine, Afrique du Sud). Dans ces derniers pays, l'absence d'obligation pour maintenir une certaine part de la sole cotonnière en coton conventionnel rend difficile aujourd'hui la poursuite des études de rentabilité comparée. Les comparaisons entre coton-Bt et coton non-Bt ont rarement été réalisées avec des variétés isogéniques (ne différant que par le seul gène $\mathrm{Bt}$ ). Cette remarque est importante dans la mesure où les différences de rendement ne sont pas uniquement dues au gène Bt et que les différences de prix ont pu être observées en défaveur du coton-Bt, car la fibre est de grade inférieur (Magaña et al., 1999) ou de longueur moindre (Orphal, op. cit.), de sorte que l'hypothèse d'une identité de prix pour les deux types de coton n'est pas toujours fondée et peut contribuer à surestimer le gain de rentabilité procuré par le coton-Bt.

\section{Efficacité et rentabilité confirmées mais variables du coton-Bt}

Avant de restituer les résultats sur l'efficacité et la rentabilité du coton-Bt, il convient de souligner la diversité des modes et des conditions de culture du coton dans le monde. Dans le domaine de la protection contre les ravageurs du cotonnier, la faible infestation (en raison des conditions climatiques locales) ne nécessite pas toujours de recourir à un 
nombre important de traitements insecticides. En Syrie, le coton peut être produit quasiment sans protection chimique. En Afrique francophone, les paysans font en moyenne cinq traitements. Dans d'autres pays, comme ce fut le cas en Amérique centrale dans les années 1980, ou en Chine dans les années 1990, on a eu à réaliser plus de vingt traitements, mais ce n'est pas une généralité. Par ailleurs, le ravageur $H$. armigera n'est pas devenu résistant aux insecticides dans tous les pays, ou dans toutes les régions d'un même pays. Le tableau 4 restitue cette diversité de situation pour comprendre la variabilité de l'efficacité et de la rentabilité du coton-Bt. Ce tableau n'est certainement pas parfait et a été difficile à constituer, car rares sont les publications qui fournissent les données de base pour déterminer les indicateurs que nous jugeons pertinents.

Tableau 4. Synthèse des gains de rentabilité procurés par le coton-Bt dans le monde

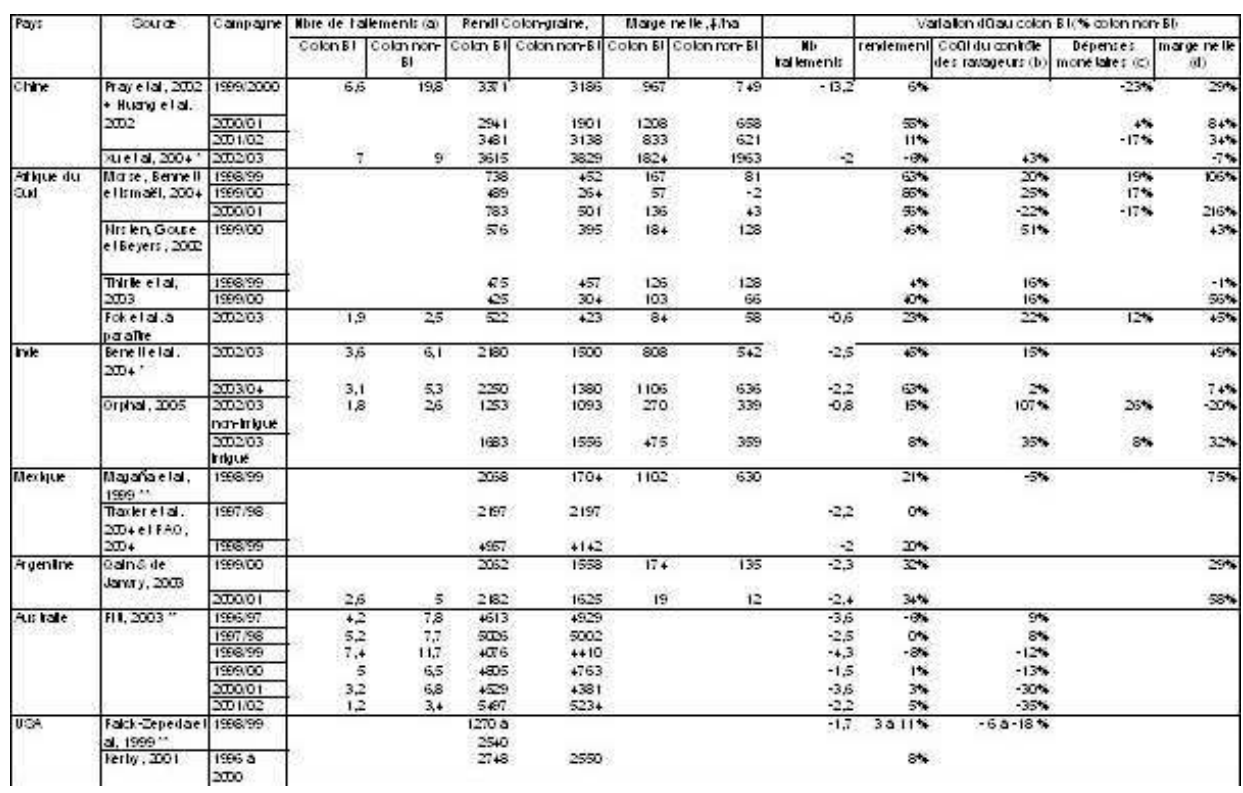

(a) Traitement contre tous les ravageurs mais les données de Pray et al. en Chine concernent seulement les traitements contre H. armigera ; (b) Coût des semences + coût des insecticides ; (c) Dépenses pour les intrants et la main-d'œuvre salariée ; (d) Marge brute-dépenses monétaires.

* Marge nette avec seulement déduction, coût, semences et insecticides.

** Variation du coût des intrants en tenant compte seulement du poste de contrôle des ravageurs (semences + insecticides).

De manière générale, l'utilisation du coton-Bt a permis de réduire le nombre de traitements insecticides contre les chenilles de la capsule du cotonnier. Cette réduction est de l'ordre de 2-4 pour la plupart des pays, mais elle est évaluée à 10-12 traitements dans le cas de la Chine (Huang et al., 2002, 2003a, 2003b, 2003c ; Pray et al., 2001, 2002). La diminution du coût lié à l'utilisation du coton-Bt est donc perçue comme très conséquente dans ce pays. Cette observation vaut cependant seulement pour les provinces de la vallée du fleuve Jaune où les résultats sont généralement rapportés et qui sont celles où la résistance des chenilles de la capsule du cotonnier avait atteint un niveau très élevé. Une communication récente relative à la province du Jiangsu, dans la vallée du fleuve Yangse, donne des résultats opposés (Xu et al., 2004) et rappelle la nécessité de se garder d'une extrapolation trop rapide des résultats des travaux du professeur Huang Jikun. En Chine, là où la pression du ravageur $H$. armigera est fluctuante et en général pas très forte, comme c'est le cas de la province du Jiangsu dans la vallée du Yangse, l'économie en traitement insecticide est faible et ne compense pas le surcoût des 
semences de coton-Bt. Par ailleurs, les variétés de coton-Bt ont montré une baisse de rendement de l'ordre de $10 \%$, parce qu'elles ont été introduites dans d'autres provinces et se sont révélées moins adaptées aux conditions locales que les variétés de coton nonBt. Ce différentiel de rendement a accentué le résultat économique négatif du coton-Bt. Ce sont des résultats dissonants qui semblent justifier les travaux que nous venons de lancer dans cette même province.

Ailleurs, il n'y a qu'aux États-Unis et en Australie où une diminution du coût est enregistrée pour le contrôle des ravageurs. En Australie, cette diminution est le résultat d'une baisse de la redevance d'emploi de 196 à 87 \$/ha au cours de la période considérée (Fitt, 2003).

Là où le traitement insecticide est réalisé au moyen de pulvériseur à dos, ce qui est le cas de la Chine et de l'Afrique du Sud, l'impact positif de la réduction du travail et de la pénibilité du travail est mis en exergue. En Chine, on a déterminé une économie en temps de travail de près de quatre-vingts jours, ce qui est fort étonnant. Il est retenu l'hypothèse qu'il faut cinq jours pour réaliser le traitement d'un hectare, ce qui est surprenant car une bonne partie des traitements est réalisée avec des pulvériseurs à dos motorisés (moins d'une demi-journée pour traiter un hectare). Cette hypothèse sur le temps de travail nous paraît aussi peu compatible avec la possibilité de traiter tous les deux jours avant l'avènement du CGM, comme cela a été évoqué. Par ailleurs, le fait de réduire le nombre de traitements contre les chenilles ne signifie pas une réduction du même ordre du nombre de pulvérisations car ces dernières associent toujours plusieurs produits visant d'autres ravageurs.

L'utilisation des semences de coton-Bt induit un surcoût qui n'est pas toujours couvert par la réduction des dépenses en insecticides. Dans beaucoup de pays, ce handicap est plus que compensé par le gain de rendement, avantage qui n'était pas vraiment pressenti au départ. Ce gain de rendement est cependant très variable entre les pays. En Australie, ce gain n'apparaît pas, mais dans un pays où la prise de conscience environnementale est forte, la réduction du nombre de traitements insecticides est considérée comme un avantage suffisant pour justifier l'emploi du coton-Bt dans une approche plus globale de production durable (Fitt et al., 2004). Aux États-Unis, ce gain de rendement est de 3 à $11 \%$ en moyenne mais avec des différences notables entre les États : là où les ravageurs cibles sont peu endémiques, surtout en année de faible niveau d'infestation, il n'y a pas de gain en rendement et la faible réduction du nombre de traitement ne justifie même pas la dépense en semences de coton-Bt. En Chine, le gain de rendement ne ressort pas nettement pour l'ensemble des régions cotonnières ayant adopté le CGM.

41 Le gain de rendement peut aussi varier entre les types de producteurs d'un même pays. Il est très élevé dans le cas des paysans Noirs de la province du Kwazulu Natal en Afrique du Sud parce que le niveau de rendement est très bas (Ismaël et al., 2002, Thirtle et al., 2003), alors qu'il est d' environ $10 \%$ chez les fermiers Blancs du même pays.

Les enquêtes pour cerner les appréciations des utilisateurs du coton-Bt mettent en évidence le sentiment de confort dans la bonne exécution du contrôle des ravageurs du cotonnier. Du côté des fermiers cultivant de grandes surfaces (États-Unis, Australie, Afrique du Sud), ils indiquent qu'ils n'ont plus à suivre aussi méticuleusement l'état de l'infestation des chenilles (par le comptage des insectes ou "scouting ») pour décider des traitements insecticides ni à craindre de ne pas pouvoir intervenir à temps en raison des conditions climatiques ou de la portance des sols. Du côté des paysans à ressources 
financières limitées et cultivant de petites surfaces, en plus de la réduction du travail et de sa pénibilité, ils soulignent l'intérêt de ne plus être soumis au risque de ne pas disposer à temps des produits insecticides.

Cette perception de l'intérêt du coton-Bt nous permet d'avancer une hypothèse sur le gain de rendement observé et sa variation. Nous pensons que le contrôle optimal des chenilles de la capsule du cotonnier est difficile voire impossible dans la pratique : on ne peut pas toujours traiter le nombre de fois qu'il faut et aux moments où les larves sont les plus sensibles aux produits épandus. Hormis les contraintes socio-économiques, telles que signalées pour le cas des paysans Noirs de l'Afrique du Sud, les pluies peuvent empêcher de traiter quand il le faudrait ou laver ce qu'on vient de pulvériser. L'éloignement du contrôle optimal est d'autant plus grand que la production est peu intensive et que le nombre de traitements réalisés était faible avant l'avènement du coton-Bt. À l'opposé, le coton-Bt libère de la toxine en permanence indépendamment des conditions climatiques ou de production du paysan, il permet donc un contrôle quasi optimal même si cela n'est pas sans inconvénient à moyen terme ${ }^{10}$. En définitive, le gain de rendement mis en évidence en faveur du coton-Bt mesure plutôt le manque à gagner lié à un contrôle chimique non optimal des chenilles, et il est normal que ce manque à gagner soit d'autant plus important que la pression des chenilles était forte et mal maitrisée par un faible nombre de traitements insecticides.

\section{Une certaine variation dans les conditions d'utilisation}

Comme la plupart des études ont été réalisées pour comparer la rentabilité suivant la méthode de budget partiel, les chercheurs impliqués retiennent l'hypothèse qu'il n'y a pas de changement d'itinéraire technique hormis la variation du nombre de traitements insecticides contre les chenilles de la capsule. Cette hypothèse est sans doute valable dans beaucoup de situations, comme aux États-Unis. En Chine aussi, il n'est pas rapporté de changement des pratiques paysannes dans l'utilisation du coton-Bt, du moins dans la vallée du fleuve Jaune. Dans la vallée du fleuve Yangse où la diffusion du CGM se fait avec des variétés hybrides et par la technique de transplantation, il pourrait en être autrement mais les travaux manquent encore pour l'attester. En Afrique du Sud, les paysans Noirs ont réduit de moitié la dose de semis pour réduire le coût à l'hectare, ce qui peut avoir un effet sur le rendement et la rentabilité. Ailleurs, en Inde et au Mexique, il est observé que les paysans semblent ajuster leurs pratiques culturales en fonction de la perception de l'espérance de rendement (Fok et al., 1999) : ils tendent à prendre davantage soin des parcelles de coton-Bt, parfois en utilisant un peu plus d'intrants, dans l'espoir de concrétiser un gain en rendement avec ce type de coton.

La pratique de la technique de prévention de la résistance, par l'installation de parcelle refuge (avec du coton conventionnel pas ou peu traité chimiquement) est rarement étudiée en tant que telle. Dans la plupart des pays, il s'agit d'un engagement contractuel des fermiers et la pratique est considérée comme acquise. En Afrique du Sud cependant, cette pratique n'est nullement respectée, du moins chez les petits paysans Noirs. En Chine, les paysans n'ont pas à s'engager contractuellement lorsqu'ils achètent des semences de CGM, ni pour ne pas utiliser les semences fermières ni pour installer une parcelle refuge. Ces particularités, pourtant importantes, ne sont pas souvent évoquées dans les publications, un seul article mentionne une relative fréquence du recours aux semences fermières en Chine. Nous savons que la stratégie de refuge n'a jamais été un 
sujet de discussion au moment du lancement du coton-Bt en Chine. Il est considéré que la petitesse des parcelles de coton ( $1 / 3 \mathrm{~d}$ 'ha) rend cette exigence peu acceptable aux yeux des paysans chinois et il semble que les techniciens chinois n'étaient pas convaincus de la pertinence de cette exigence dans un contexte de co-habitation de nombreuses cultures, toutes hôtes potentielles de H. armigera.

Presque toutes les études comparent la rentabilité avec le coton-Bt introduit, principalement sur l'initiative de Monsanto. Il est rarement fait mention de variétés de CGM concurrentes créées localement. Les articles sur l'expérience chinoise font exception en évoquant parfois l'offre de variétés chinoises, mais leur origine, leur part de marché et l'évolution de celle-ci ne sont pas analysées.

La plupart des études prennent les conditions d'utilisation comme des données figées et non comme sujets d'analyse. La différence de ces conditions entre la Chine et les ÉtatsUnis, au regard notamment du paiement ou non de la redevance d'emploi du CGM, explique fondamentalement la répartition différente de la valeur ajoutée découlant de l'utilisation du CGM. Aux États-Unis où la redevance d'emploi est de plus de $70 \$ /$ ha, ce sont les firmes de biotechnologies et de distribution semencière qui accaparent la plus grosse part de la valeur ajoutée alors qu'en Chine, les paysans en sont les principaux bénéficiaires (Falck-Zepeda et al., 1999, 2000; Taxler et al., 1999). C'est dans les publications plus récentes où la question de l'efficacité du niveau de la redevance d'emploi est posée (Qaim et al., 2003). Il est observé que le niveau exigé parait élevé et qu'il freine ainsi l'extension de la diffusion de l'utilisation du CGM ou encourage les comportements de cavalier libre. Cette observation est explicitement reprise dans le récent rapport d'un panel international sur les perspectives du CGM dans le monde (ICAC, 2004). La démarche de gestion flexible de la redevance d'emploi du CGM n'a pas fait l'objet de réflexion spécifique alors qu'elle a sans doute contribué à la poursuite de son utilisation en Australie.

En résumé, les expériences d'utilisation du coton-Bt ont donné partout des résultats globalement positifs. L'expérience du CGM en Chine tend à être présentée comme un exemple de succès d'utilisation par les petits paysans à suivre pour les autres PVD (Huang et al., 2002). C'est une conclusion qui donnerait ainsi raison à l'optimisme de nombre d'observateurs (Lague, 2002 ; McGloughlin, 1999; Prakash, 2004 ; Shelton, 2003) et qui justifierait les actions de promotion associant des organismes de charité (Hoag, 2003) afin que l'Afrique ne souffre plus du manque à gagner que certains auteurs ont évalué pour le Mali (Cabanilla et al., 2003).

L'expérience du CGM en Chine est certes déjà rapportée par plusieurs articles. Les travaux que nous avons conduits récemment présentent un aspect complémentaire en permettant d'appréhender la perception des paysans utilisateurs et de resituer les conditions d'introduction du CGM afin de souligner les facteurs institutionnels qui ont favorisé son adoption.

\section{Résultats d'adoption du CGM dans un contexte institutionnel favorable}

50 Il n'y a pas de recensement officiel des statistiques relatives à l'utilisation du CGM en Chine, les estimations fondées sur les travaux de terrain permettent de dire que l'adoption du CGM est proche de $100 \%$ là où cette utilisation est effective et a permis une 
relance de la production (cas de la province du Hebei). À l'échelle de la Chine, on peut avancer que plus de $40 \%$ de la surface cotonnière chinoise sont couverts par du CGM (tableau 5), car la principale zone de production, dans la province du Xinjiang, n'est pas encore concernée.

Tableau 5. Evolution de la surface en CGM en Chine $\left(10^{3}\right.$ ha)

\begin{tabular}{c|c|c|c|c|c|c|} 
& \multicolumn{3}{|c|}{ Toutes provinces } & \multicolumn{3}{c|}{ Province du Hebei } \\
\cline { 2 - 7 } & Tout coton & CGM & $\%$ CGM & Tout coton & CGM & $\%$ CGM \\
\hline 1997 & 4491 & 34 & $1 \%$ & 377 & 13 & $3 \%$ \\
\hline 1998 & 4459 & 261 & $6 \%$ & 315 & 175 & $56 \%$ \\
\hline 1999 & 3726 & 654 & $18 \%$ & 266 & 227 & $85 \%$ \\
\hline 2000 & 4041 & 1216 & $30 \%$ & 307 & 298 & $97 \%$ \\
\hline 2001 & 4800 & 2174 & $45 \%$ & 418 & 410 & $98 \%$ \\
\hline 2002 & 4480 & 1943 & $43 \%$ & 407 & 403 & $99 \%$ \\
\hline
\end{tabular}

Sources: Huang Jikun et al., 2003a et compilation par les auteurs à la prescription courante de mise en place de parcelles refuges pour prévenir la résistance des ravageurs au CGM. Cette prescription n'a pas lieu en Chine, et en raison de la petitesse des parcelles, de la situation de polyculture et du caractère très polyphage (grand nombre de plantes hôtes) de H. armigera, elle ne semble pas nécessaire (Wu, 2002 ; Wu et al., 2004b).

commercialisation de CGM pour utilisation par de « petits paysans » apparaît donc tout à fait réussie. Nous pouvons l'illustrer à partir des résultats de l'enquête que nous avons conduite en 2003. Cette enquête a été réalisée pour cerner les pratiques de culture et les résultats économiques en relation avec la structure des exploitations agricoles et la maîtrise du contrôle des ravageurs. L'enquête a couvert les cinq comtés les plus cotonniers de la province du Hebei (Cangzhou, Handan, Hengshui, Shijiazhuang and Xingtai), à raison d'un ou de deux villages par comté. Un ensemble de plus de 200 exploitations a été interviewé en trois à quatre passages dans huit villages, dont deux sont partiellement ou totalement impliqués dans la production semencière (en particulier le village de Liang Mian Chang dont nous n'utilisons pas toujours les résultats). La taille des exploitations enquêtées est représentative des exploitations chinoises, en moyenne moins d'un hectare, sauf pour les villages semenciers où les paysans peuvent accéder aux terres des fermes semencières (tableau 6). En raison du peu de différence entre les villages enquêtés, les résultats seront présentés pour l'ensemble de l'échantillon.

\section{Efficacité et rentabilité dans un contexte d'offre concurrentielle de variétés}

\section{CGM efficace...au moins à court terme}

53 L'efficacité du CGM, avec les variétés de coton-Bt se révèle par le niveau élevé et peu variable du rendement en coton-graine (tableau 6), même si une baisse a été observée en 2003 et attribuée en général aux pluies excessives en fin de saison.

L'efficacité de l'utilisation du CGM découle de la réduction du nombre de traitements insecticides qui pouvait atteindre 25 à 30 traitements (Zhang et al., 2003). Dans notre 
enquête, ce nombre a varié de 10 à 12 (tableau 7), dont 5-6 contre H. armigera, ce qui est conforme aux résultats des travaux antérieurs (Huang et al., 2003a ; Pray et al., 2002).

Tableau 6. Données générales sur les villages enquêtés

\begin{tabular}{|c|c|c|c|c|c|c|}
\hline \multirow[t]{2}{*}{ Village } & \multirow{2}{*}{$\begin{array}{l}\text { Nombre } \\
\text { paysans } \\
\text { enquêtés }\end{array}$} & \multirow{2}{*}{$\begin{array}{c}\text { Nombre } \\
\text { membres } \\
\text { par famille }\end{array}$} & \multicolumn{2}{|c|}{$\begin{array}{l}\text { Surface cultivée par } \\
\text { paysan enquêté (ha) }\end{array}$} & \multicolumn{2}{|c|}{$\begin{array}{l}\text { Rendement coton-graine des paysans } \\
\text { enquêtés, moyenne et écart-type,kg/ha }\end{array}$} \\
\hline & & & totale & coton & 2002 & 2003 \\
\hline Chen Zhuang & 29 & 4,7 & 0,91 & 0,81 & $3625(233)$ & $3032(444)$ \\
\hline DaWangZhuang & 26 & 4,0 & 0,71 & 0,23 & $3914(201)$ & \\
\hline Fang $\mathrm{Ya}$ & 26 & 4,0 & 0,69 & 0,21 & $3424(261)$ & $3346(173)$ \\
\hline ShiJiaBao & 33 & 4,3 & 0,79 & 0,34 & 4204 (399) & $3691(378)$ \\
\hline Song Zuang & 25 & 4,0 & 0,66 & 0,32 & $3876(219)$ & $3763(75)$ \\
\hline XiGuan & 38 & 3,5 & 0,97 & 0,20 & $2855(436)$ & \\
\hline XiJing $\mathrm{Ke}$ & 30 & 4,5 & 0,59 & 0,37 & $3546(398)$ & $3015(462)$ \\
\hline Echantillon & 207 & 4,2 & 0,76 & 0,35 & & \\
\hline
\end{tabular}

Tableau 7. Nombre total de traitements insecticides

\begin{tabular}{l|c|c}
\hline Nbres moyens de traitements & $\mathbf{2 0 0 2}$ & $\mathbf{2 0 0 3}$ \\
\hline Contre Aphis & 4,1 & 5,2 \\
\hline Contre H. arrigera & 5,7 & 4,6 \\
\hline Contre araignées rouges & 2,7 & 3,3 \\
\hline Contre tous les ravageurs* & 10,1 & 12,1
\end{tabular}

* Certaines exploitations n'ont pas traité contre les aphis ou les araignées, les moyennes ne sont pas calculées avec le même nombre d'individus, la moyenne du nombre total de traitements est ainsi légèrement différente du total des moyennes.

Lorsqu'on décompose les traitements insecticides en fonction des ravageurs visés, il ressort que les paysans traitent encore cinq fois contre $H$. armigera, mais ce nombre est dépassé par celui des traitements réalisés pour contrôler les autres ravageurs (tableau 7).

Les paysans enquêtés ont exprimé leur satisfaction sur l'efficacité des variétés de coton Bt car ils savent presque tous que ces variétés sont seulement efficaces contre H. armigera (93\%). Néanmoins notre enquête révèle qu'ils divergent sur la perception de l'évolution du complexe des ravageurs. Un tiers d'entre eux voit davantage d'espèces de ravageurs, un autre tiers affirme que la pression parasitaire est devenue moindre alors que le dernier tiers n'a pas observé de changement. Cependant, les paysans ne sont pas très optimistes sur la durée de l'efficacité du CGM : sur les 200 paysans qui ont donné leur avis, 86 déclarent avoir vu une certaine perte d'efficacité contre H. armigera, et pour les autres, $70 \%$ d'entre eux pensent que la résistance de H. armigera contre le CGM surviendra.

Face à ce risque qui menace la durabilité de la solution CGM, des actions sont mises en œuvre, comme la combinaison de gènes à actions diverses dans les nouvelles variétés (par exemple des gènes d'inhibition de protéase en complément des gènes $\mathrm{Bt}$ ). Ces actions peuvent cependant s'avérer insuffisantes si le danger provient d'un changement du complexe des ravageurs : ce changement peut se traduire par une importance croissante des espèces plus difficiles à contrôler, surtout dans la situation d'une absence totale de coordination entre les paysans chinois pour contrôler les attaques des ravageurs. C'est une menace que certains chercheurs chinois rencontrés prennent au sérieux. Depuis ces dernières années, il est observé qu'une chenille très vorace et très polyphage, Spodoptera litoralis ou Prodenia litura (Fabricius), est devenue très présente sur le cotonnier (Li, 2004). En raison de cette menace, il convient donc de prendre le succès chinois tel qu'il est, c'est- 
à-dire une efficacité à court terme qui ne présente pas encore de garantie suffisante pour perdurer.

\section{CGM à prix accessible}

Les paysans chinois ont accédé au CGM à un coût bien moindre que leurs homologues dans d'autres pays : cela est la conséquence des règles de diffusion en Chine que nous avons déjà brièvement évoquées. Le tableau 8 montre le prix moyen des semences, du moins pour les paysans qui les ont achetées. Les semences des variétés chinoises, quand il ne s'agit pas de variétés hybrides, sont environ $30 \%$ moins chères que les variétés américaines introduites dès 1998. Même les variétés hybrides chinoises, proposées récemment et d'utilisation encore peu courante dans la zone enquêtée, ont un prix compétitif face aux variétés américaines. Après conversion en dollar américain, les semences de CGM coûtent en moyenne moins de 60 US \$/ha en 2003 (environ 40 US \$/ha pour les variétés chinoises). Ce montant est inférieur au seul coût de la redevance d'emploi que les paysans ont à payer dans les autres pays (en moyenne 60-90 US $\$ / \mathrm{ha}$ ).

Tableau 8. Prix et parts de marché des variétés d'origine américaine et chinoise

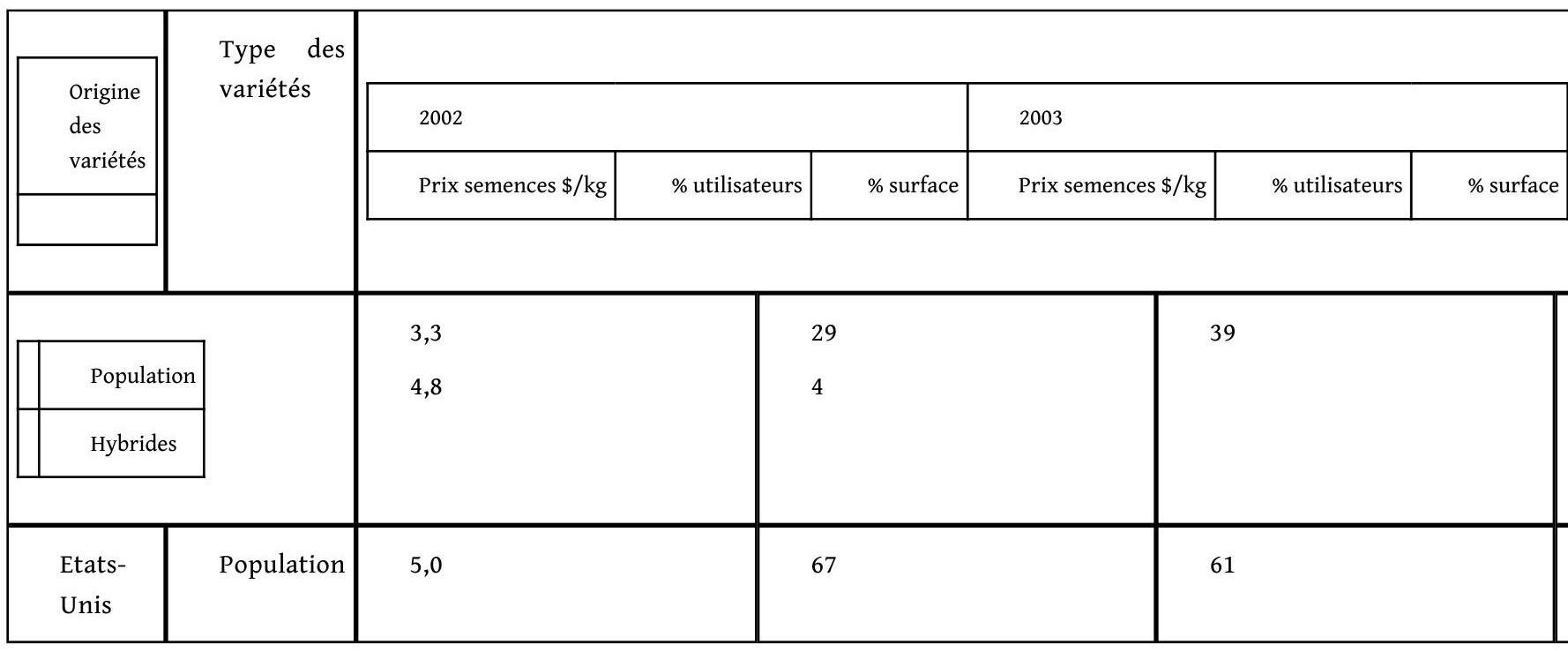

Source : notre enquête.

L'accessibilité du CGM peut être encore mieux cernée en termes relatifs. Le passage à l'utilisation du CGM a induit une forte augmentation du coût des semences qui n'était que d'environ $0,25-0,35$ US $\$ / \mathrm{kg}$ avec les variétés de coton conventionnel. Néanmoins, l'augmentation du coût, certes de dix à vingt fois, ramène seulement les semences de coton à un niveau du coût auquel les paysans avaient été habitués pour l'utilisation généralisée de semences hybrides de maïs. Par ailleurs, dans un contexte de culture fortement intensive en intrants, le nouveau coût des semences correspond seulement à un tiers du coût total des produits chimiques (engrais et pesticides confondus), il s'agit donc d'une augmentation dans une proportion qui peut passer pour acceptable.

Plus important à considérer est la possibilité des paysans de ne pas avoir à acheter les semences de manière systématique, grâce au droit préservé d'utiliser les semences fermières issues de leurs propres productions. Il en découle que le coût réel des semences 
est bien moindre que celui ressortant du tableau précédent : certains paysans dans notre enquête mentionnent en effet un coût nul pour les semences. Globalement, moins de la moitié des paysans enquêtés déclarent acheter en totalité leurs semences (tableau 9).

Tableau 9. Phénomène fréquent d'utilisation de semences fermières issues de la production propre des paysans

\begin{tabular}{|c|c|c|c|c|}
\hline & \multicolumn{3}{|c|}{ Paysans en fonction du nombre de variétés cultivées } & \multirow[t]{2}{*}{ Tous les paysans } \\
\hline & 1 & 2 & 3 & \\
\hline Semences obtenues par échange & $1 \%$ & $0 \%$ & $0 \%$ & $1 \%$ \\
\hline Semences partiellement achetées & $26 \%$ & $29 \%$ & $75 \%$ & $30 \%$ \\
\hline Semences achetées en totalité & $53 \%$ & $33 \%$ & $0 \%$ & $45 \%$ \\
\hline Semences du paysan en totalité & $20 \%$ & $38 \%$ & $25 \%$ & $25 \%$ \\
\hline Total & $100 \%$ & $100 \%$ & $100 \%$ & $100 \%$ \\
\hline
\end{tabular}

Source : enquête

\section{Une situation de marché concurrentiel}

Le niveau très acceptable du coût des semences de CGM provient de l'instauration d'une forte concurrence ${ }^{11}$, cela est opposé au marché monopolistique prédit par de nombreux opposants à l'utilisation d'OGM dans les PVD. Dans notre enquête, nous avons recensé 28 variétés, un nombre plus grand quoique conforme à celui évoqué par d'autres travaux (Huang et al., 2003b ; Pray et al., 2001), alors qu'il y a six variétés dont nous n'avons pas pu faire préciser les noms et qui ne sont pas forcément des variétés distinctes ${ }^{12}$.

Non seulement il n'y a pas de marché monopolistique des semences de CGM en faveur des firmes internationales qui ont introduit les premières variétés, il ressort aussi que la capacité de mise en marché de variétés de CGM par les institutions locales de recherche a été accrue, aux différents niveaux administratifs (tableau 10) contestant ainsi la suprématie initiale des variétés américaines. Dans la campagne 2002, la part de marché des variétés américaines restait supérieure, en termes de nombre de paysans utilisateurs et de surface couverte, mais ce n'est plus le cas à la campagne suivante (cf. tableau 8).

Tableau 10. Nombre de variétés CGM en fonction des niveaux administratifs d'obtention et des types de variété

\begin{tabular}{l|c|c|c}
\hline \multirow{2}{*}{ Niveau administratif d'obtention } & \multicolumn{3}{|c}{ Type de variétés } \\
\cline { 2 - 4 } & Populations & Hybrides & Total \\
\hline International & 2 & & 2 \\
\hline National & 9 & 1 & 10 \\
\hline Provincial & 4 & 1 & 5 \\
\hline District & 2 & 3 & 5 \\
\hline Indéterminé & 6 & & 6 \\
\hline Total & 23 & 5 & 28 \\
\hline
\end{tabular}

Source : notre enquête.

\section{Une utilisation rentable}

Produire du coton se révèle être rentable et attractif, du moins en termes monétaires avant la prise en compte de la quantité et de la pénibilité du travail. En comparaison du maïs et du blé qui sont les cultures alternatives et qui sont le plus souvent cultivés en relais (maïs installé peu avant la récolte du blé), le revenu du coton est supérieur aux 
revenus cumulés des deux céréales, que ce soit en revenu brut ou net des coûts des intrants (tableau 11). La rentabilité du coton est même plus importante en 2003 en conséquence d'une remontée du prix du coton qui a plus que compensé la diminution du rendement. La rentabilité financière de la culture du coton est davantage mise en exergue par le niveau élevé du ratio output/input, de l'ordre de 6 à 7, bien supérieur au seuil de 3 communément admis et qui correspond d'ailleurs à ce qui est obtenu sur le blé et le maïs.

Tableau 11. Comparaison des revenus des cultures de coton, blé et maïs (US \$/ha)

\begin{tabular}{|l|cc|cc|}
\hline & \multicolumn{2}{|c|}{ Revenu brut } & \multicolumn{2}{c|}{ Revenu net des coûts des intrants } \\
\cline { 2 - 5 } & 2002 & 2003 & 2002 & 2003 \\
\hline Coton & 1716 & 2377 & 1425 & 2064 \\
B1é & 781 & 819 & 461 & 554 \\
Mais & 814 & 880 & 578 & 707 \\
\hline
\end{tabular}

Source : notre enquête

\section{Place des facteurs institutionnels dans le succès chinois}

Le succès de la diffusion du CGM en Chine procède de facteurs techniques, économiques et institutionnels. Le CGM a apporté une réponse technique à un problème sérieux. Son utilisation est devenue plus rentable grâce à un contexte économique favorable alors que sa diffusion a bénéficié de règles spécifiques que la Chine a su imposer, même face à des firmes multinationales.

\section{Conditions économiques favorables à une solution technique fortement attendue}

Comme nous l'avons indiqué dès l'introduction, la progression de la production cotonnière dans la province du Hebei a été arrêtée par l'apparition de la résistance de $H$. armigera aux insecticides. La commercialisation du CGM a manifestement offert une solution qui a permis d'inverser la tendance de la production cotonnière trois ans après la commercialisation du CGM (cf. figure 1).

Evolution de la production cotonnière, Province du Hebej

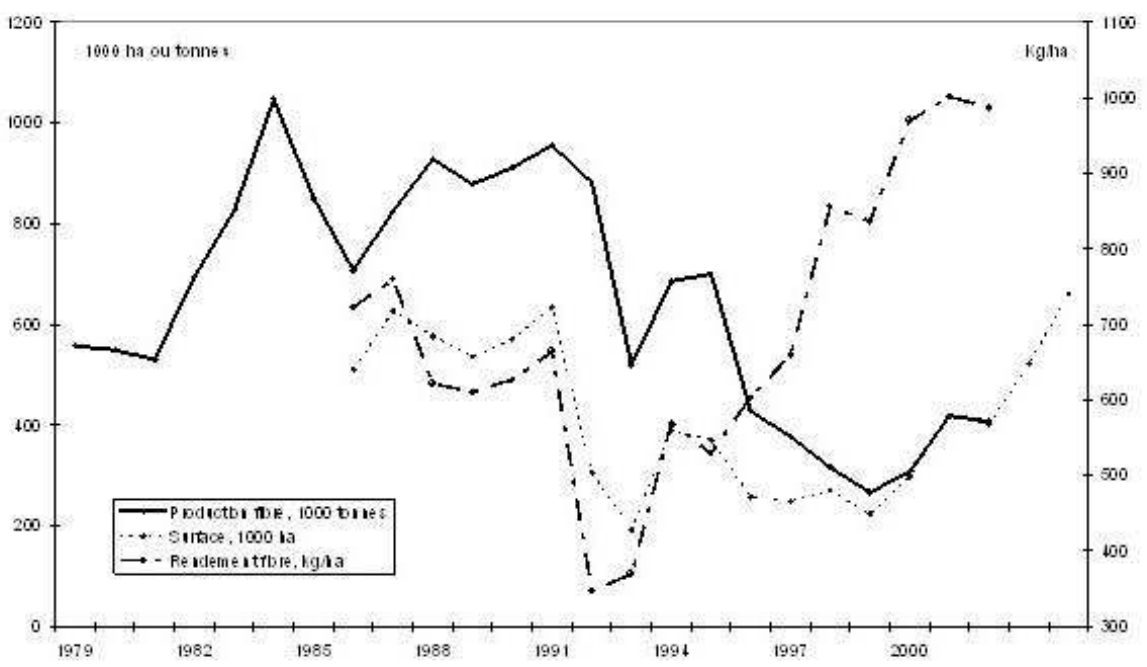


66 augmentation limitée des coûts de production et une rémunération satisfaisante du coton, que ce soit en termes absolus ou en termes relatifs.

eau des coûts de production, nous avons déjà souligné que, en termes absolus, le coût d'utilisation de semences CGM est nettement inférieur à celui observé dans d'autres pays, et ce d'autant plus que les paysans chinois sont les rares paysans au monde à disposer du droit d'utiliser les semences venant de leurs propres productions.

En termes relatifs, le coût des semences de CGM est seulement équivalent à celui consenti depuis très longtemps par les paysans pour cultiver les variétés hybrides de maïs dont ils doivent renouveler les semences tous les ans. Par ailleurs, dans une situation de production intensive comme nous l'avons déjà souligné, le surcoût des semences ne représente que le tiers du coût total des facteurs monétaires de production et peut paraitre ainsi tout à fait acceptable.

69 Au niveau du prix obtenu pour la vente du coton, les paysans bénéficient également de conditions favorables, tant en termes absolus que relatifs. L'entrée à l'OMC a certes conduit la Chine à supprimer toutes les subventions aux facteurs de production, à cesser de soutenir directement le prix et à accepter la détermination de quota d'importation à tarif préférentiel de $1 \%$, mais on observe que les paysans chinois bénéficient d'un prix supérieur à ce qu'aurait permis la seule répercussion du prix mondial ${ }^{13}$ (figure 2) Cette situation favorable procède du maintien du contrôle des importations qui permet d'isoler encore quelque peu les paysans chinois du marché mondial ${ }^{14}$. De plus, elle réduit le risque financier de la culture cotonnière relancée par l'utilisation du CGM.

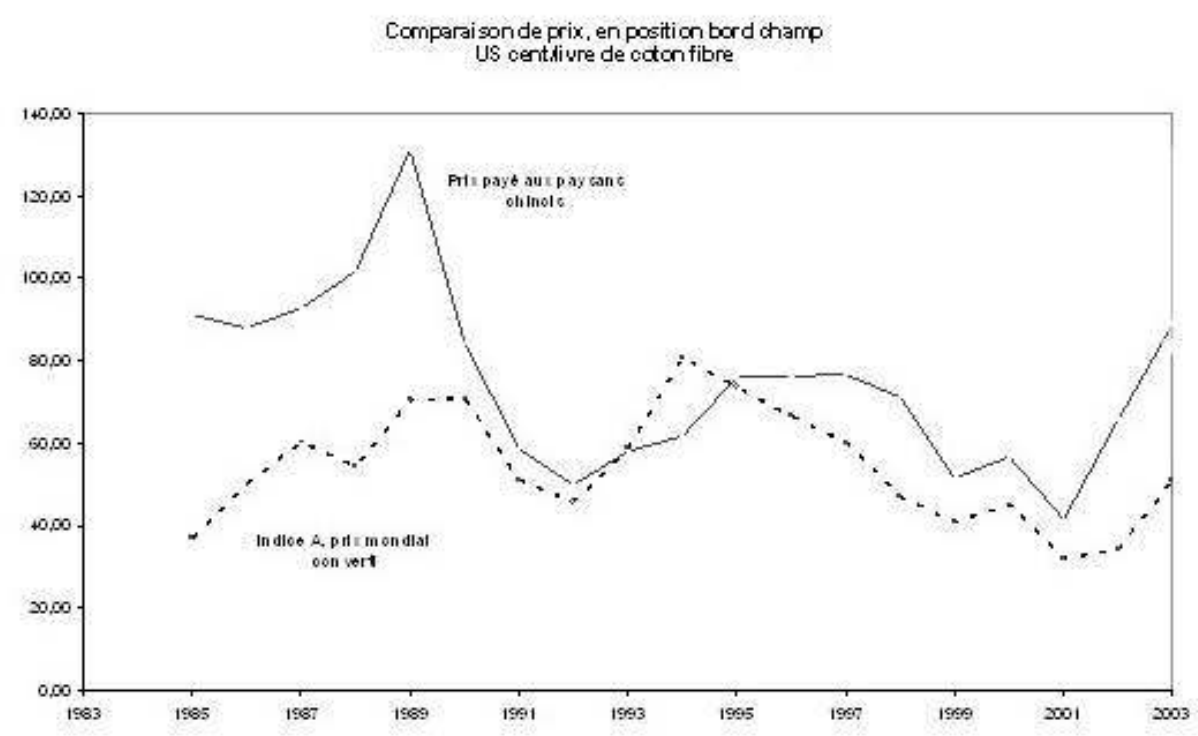

\section{Innovation institutionnelle propice à une large et rapide diffusion du CGM}

Bien que la réelle commercialisation du CGM en 1998 ait impliqué des multinationales américaines qui avaient déjà établi des règles d'utilisation dans d'autres pays, les règles qui ont été retenues en Chine sont très différentes et elles sont bien plus profitables aux paysans. Pour cette raison, à l'opposé des travaux précédents, nous ne pensons pas que la diffusion du CGM a dépendu uniquement de ses qualités intrinsèques et nous croyons que 
les règles accompagnant cette diffusion ont compté davantage dans la rentabilité d'utilisation et dans l'émergence d'un marché contestable.

Les règles de commercialisation du CGM dans le monde ont été fixées par la multinationale Monsanto, d'abord aux États-Unis, puis étendues ensuite à d'autres pays, à savoir l'Australie, l'Argentine, l'Afrique du Sud... Monsanto, avec son allié semencier Delta and Pineland Company, a été la première société à commercialiser le coton-Bt dans le monde. Tous deux jouissent jusqu'à ce jour d'une situation de quasi-monopole.

Dans la commercialisation des semences de CGM, le prix des semences n'est pas réellement différent de celui des semences de coton conventionnel. Ce qui est nouveau, c'est l'exigence d'un paiement de redevance d'emploi d'une part, et l'engagement contractuel des paysans à ne pas utiliser les graines de leur production pour servir de semences et à se conformer aux techniques de prévention de la résistance des ravageurs aux toxines Bt par installation de parcelle refuge, d'autre part.

Une autre caractéristique de l'approche mondiale dans la diffusion du CGM est la promotion d'un nombre limité de variétés. En Afrique du Sud, les variétés NuCotton et NuOpal ont été successivement lancées. En Chine, ce fut le cas pour les variétés 33B et 99B qui ont été commercialisées aussi au Mexique.

La Chine a réussi à faire appliquer des règles totalement différentes. Elle réussit à faire valoir des règles de commercialisation qui prévinrent l'émergence d'une situation de monopole et d'une implication exclusive des firmes américaines. En 1996, ces firmes furent invitées à se joindre à la Hebei Seed Company pour créer une société d'économie mixte alors qu'une autre société chinoise, impliquant l'Académie des sciences agricoles de Chine fut installée. L'autorisation à la commercialisation du CGM était limitée d'abord à trois provinces avant d'être étendue à neuf provinces. L'utilisation massive même dans des provinces sans autorisation officielle, comme c'est le cas du Jiangsu dans la vallée du fleuve Yangse, la diffusion d'un grand nombre de variétés émanant d'institutions de recherche de divers niveaux administratifs, et le phénomène d'utilisation de semences fermières permettent de dire que la situation initiale de duopole au début de la commercialisation du CGM est aujourd'hui bien révolue.

La fourniture de semences de coton-Bt est réalisée suivant des modalités qui sont similaires à celles prévalant dans les pays occidentaux pour les variétés conventionnelles, sans paiement de redevance d'emploi et sans engagement contractuel des paysans, ni pour suivre des techniques particulières de culture, ni pour s'interdire l'usage de semences fermières.

Dans le domaine de l'approvisionnement en semences de coton en Chine, on peut tout de même dire que ces modalités furent nouvelles sinon révolutionnaires. La commercialisation des semences de coton-Bt a permis d'exprimer, sur l'initiative de la société d'économie mixte impliquant les firmes américaines, une offre d'un service moderne de semences, avec la garantie de semences de qualité traitées chimiquement pour assurer une germination parfaite et une forte vigueur des plantules. Tout cela est révélé par un packaging spécifique et attractif contenant les instructions aux utilisateurs et les indications pour se référer au service clientèle. Lorsque des produits de contrefaçon firent leur apparition, on ajouta un codage, sorte de numéro de série, pour permettre aux clients de vérifier, en téléphonant à un numéro vert, s'ils ont été abusés ou pas. Il s'agit d'une innovation dans le domaine semencier qui est aujourd'hui captée par de 
nombreuses sociétés chinoises et dont l'impact positif va au-delà du seul coton-Bt, voire de la seule culture du coton.

\section{Conjonction de facteurs difficile à répliquer}

Comme pour toute innovation, il faut du recul pour affirmer son succès. Dans le cas particulier du CGM, bien que les résultats de court terme paraissent convaincants, il reste difficile d'anticiper les effets à moyen et long terme. Le complexe des ravageurs semble être en évolution depuis l'utilisation du CGM, elle peut se traduire par des dégâts plus importants de la part de ravageurs qui pourraient être plus difficiles à contrôler. Cette réserve faite, nous pensons qu'il est pertinent d'analyser les facteurs à la base du « succès » chinois, au-delà des règles de diffusion que le gouvernement chinois réussit à faire valoir. Cela nous paraît utile pour cerner les possibilités d'étendre l'expérience chinoise dans d'autres pays, notamment les PVD.

\section{Identification des facteurs favorables en Chine}

Pour ce qui concerne l'adoption par les paysans, nous devons rappeler l'attente, implicite sinon explicite, d'une solution technique pour aider à surmonter un phénomène de résistance de $H$. armigera et relancer la production cotonnière dans plusieurs provinces. La réponse positive et rapide à la commercialisation du CGM n'est donc pas étonnante.

S'agissant du résultat économique de l'adoption du CGM, deux facteurs liés concourent à rendre la culture cotonnière davantage rentable. Le haut niveau de rendement accentue l'impact positif d'un prix d'achat plus favorable du coton produit.

Le niveau élevé du rendement obtenu en Chine procède d'une forte intensification dans l'utilisation des intrants (engrais, pesticides...). Il en découle que le coût additionnel lié à l'utilisation des semences de CGM peut paraître alors tout à fait acceptable, car son effet sur l'augmentation du coût total des intrants reste limité. En d'autres termes, le CGM a consisté en une nouvelle étape d'une intensification déjà fort poussée.

Plusieurs facteurs nous semblent être à l'origine de la diversité des variétés proposées de CGM et de leur mise en concurrence (avec effet positif pour les paysans). D'abord, la Chine possède sa propre construction de gène $\mathrm{Bt}$ dont l'utilisation peut être gérée indépendamment de la stratégie de toute multinationale. Ensuite, les scientifiques chinois sont parvenus à une grande maitrise dans le domaine du transfert de gène, soit avec les techniques habituelles soit avec une technique qui leur est propre. En troisième lieu, après le lancement d'un programme ambitieux depuis le milieu des années 1980, la Chine possède un portefeuille conséquent de gènes d'intérêt agronomique de sorte que la combinaison de plusieurs gènes, dans de nouvelles variétés, peut conduire à une efficacité supérieure à celles de la première génération de coton Bt. En fait, ce réservoir de gènes spécifiques s'ajoute à un portefeuille très garni de variétés de coton, constitué en plusieurs décennies par des institutions de recherche, relevant de divers niveaux administratifs, toutes désireuses de saisir l'opportunité des biotechnologies. Ces deux derniers facteurs, large gamme de variétés disponibles et grand nombre d'institutions de recherche motivées, est à la base de la rapide mise en marché de nombreuses variétés de CGM. 
Dans le domaine des règles de diffusion du CGM, nous avons souligné le rôle joué par l'État. Ce rôle peut être décomposé en plusieurs facteurs favorables. D'abord, il doit y avoir une volonté de l'État à s'impliquer. Ensuite, cet État doit disposer d'un pouvoir de négociation face aux firmes multinationales pour leur faire accepter des règles s'écartant de celles qu'elles appliquent généralement. Il doit y avoir également une politique générale de gestion des investissements directs étrangers de manière que les entreprises nationales puissent bénéficier de l'introduction de technologie nouvelle. Enfin, une certaine connaissance des contraintes et des pratiques paysannes est nécessaire pour ajuster les règles de diffusion dans un sens favorable aux paysans.

Pour assurer un prix attractif aux producteurs de coton, une certaine protection que la Chine réussit à maintenir a été déterminante.

Globalement, par-delà les règles de commercialisation que le gouvernement chinois parvint à mettre en place, nous trouvons en fait une combinaison d'un grand nombre de facteurs ou de conditions favorables dans les domaines technique et institutionnel. C'est ce que nous avons résumé dans la première colonne du tableau 12. La question se pose de savoir s'il est possible de retrouver une telle combinaison favorable dans d'autres pays.

Tableau 12. Facteurs favorables à la diffusion du CGM

\begin{tabular}{|c|c|c|c|c|}
\hline \multirow[t]{2}{*}{ Facteurs favorables } & \multicolumn{4}{|c|}{ Rencontrés? } \\
\hline & Chine & Inde & Brésil & SSA \\
\hline $\begin{array}{l}\text { Niveau élevé de résistance des ravageurs aux insecticides } \\
\text { menaçant la poursuite de la production }\end{array}$ & Oui* & Oui & Non & Non \\
\hline Niveau élevé de rendement & Oui & Non & Oui** & Non \\
\hline Niveau élevé d'intensification en intrants & Oui & Non & Oui** & Non \\
\hline Disponibilité de constructions de gène $\mathrm{Bt}$ & Oui & Oui? & Non & Non \\
\hline Bonne maitrise en biotechnologies & Oui & Oui & Oui & Non \\
\hline $\begin{array}{l}\text { Portefeuille garni en gènes d'intérêt agronomique et } \\
\text { d'origine national }\end{array}$ & Oui & Oui? & Non & Non \\
\hline Portefeuille garni en variétés de coton & Oui & Oui & Oui & Oui \\
\hline Grand nombre d'institutions de recherche & Oui & Oui & Oui & \pm Oui \\
\hline $\begin{array}{l}\text { Volonté de l'Etat à s'impliquer dans la définition des règles } \\
\text { de commercialisation des semences }\end{array}$ & Oui & Non & Non & \pm Oui \\
\hline Bon pouvoir de négociation & Oui & \pm Non & \pm Non & Non \\
\hline $\begin{array}{l}\text { Politique de gestion des investissements étrangers au profit } \\
\text { des entreprises nationales }\end{array}$ & Oui & \pm Non & \pm Non & Non \\
\hline $\begin{array}{l}\text { Connaissance et capacité à ajuster les règles de } \\
\text { commercialisation au profit des petits paysans }\end{array}$ & Oui & Non & Non & Non \\
\hline $\begin{array}{l}\text { Capacité à assurer un prix favorable dans l'achat du coton } \\
\text { produit par les paysans }\end{array}$ & oui & \pm Non & Non & Non \\
\hline
\end{tabular}

* dans les provinces de la vallée du fleuve Jaune, c'est moins le cas dans la vallée du fleuve Yangse ** appréciation valable pour les fermes commerciales, pas forcément pour les petits paysans, peu impliqués dans la production cotonnière au Brésil. 


\section{Réplication peu envisageable des facteurs favorables par les seules forces du marché} position. Ce pays a récemment fait savoir qu'il disposait de sa propre construction de gène Bt. Mais même dans ce pays, et c'est aussi valable pour le Brésil, on ne rencontre pas la plupart des facteurs positifs qui ont eu une influence positive en Chine, en raison notamment de l'orientation libérale de leur économie qui écarte toute intervention de l'État. Il nous parait donc bien difficile d'affirmer que les résultats économiques du CGM, obtenus aujourd'hui par les paysans chinois, seront ce que les paysans d'autres pays auront.

\section{Défis pour un partenariat public/privé rénové et une réhabilitation de l'aide à l'intensification}

Il n'y a cependant pas de fatalité à la vision pessimiste que nous dégageons si des évolutions positives peuvent se concrétiser dans les domaines de la propriété intellectuelle ou de l'appui au développement.

Un très grand nombre de brevets couvrent la propriété des gènes et des procédés de transformation et d'introgression dans le génome (Kowalski et al., 2002). La contrainte et le coût du respect de cette propriété sont reconnus comme les freins à la diffusion des variétés génétiquement modifiées, justifiant ainsi les appels à leur assouplissement (ICAC, 2004 ; Taylor et al., 2003). Les analyses restent fréquemment attachées au respect intangible du droit de propriété dans sa forme actuelle. À l'opposé de ce qui a été obtenu dans le domaine pharmaceutique, il y a peu de perspective d'évolution en raison de la divergence des opinions sur l'intérêt des OGM en agriculture. 
90 D'un autre côté, le respect des brevets pourrait s'avérer moins contraignant à brève échéance du fait qu'un grand nombre de brevets devrait tomber dans le domaine public. Cette réalité proche semble justifier la pertinence à établir un processus, avec implication d'organismes régionaux ou internationaux, pour aider les PVD à identifier les techniques et produits biotechnologiques facilement accessibles et à faible coût (Pingali et al., 2004). Par un tel processus, les organismes publics de recherche dans les PVD pourront ainsi accéder aux produits biotechnologiques pour les insérer dans du matériel génétique localement adapté, comme ce fut le cas dans le cadre de la révolution Verte, avant l'avènement des variétés génétiquement modifiées. Un partenariat public/privé pourrait ainsi émerger de nouveau, en laissant le secteur privé investir l'activité de production de semences. Le fait que des pays comme l'Inde et la Chine aient abouti à des résultats notables de biotechnologie, à partir de financement public, signifie aussi une dépendance moins exclusive des PVD vis-à-vis des seules multinationales pour la fourniture de produits et de procédés en biotechnologie. C'est une situation nouvelle qui peut être favorable à l'obtention de conditions meilleures d'utilisation, à condition que les PVD ne soient pas laissés seuls dans leur face-à-face avec les multinationales et soient assistés par des acteurs internationaux pouvant être de nature diverse (ONG, organisations internationales...).

91 L'adoption effective des OGM en agriculture dépend aussi des conditions économiques d'intensification que l'application des Plans d'ajustement structurel a largement sapées pendant deux décennies dans les PVD. Dans le cadre des négociations du Cycle de Doha, l'idée du soutien aux paysans à faibles ressources financières semble être réhabilitée aujourd'hui (Fok, 2002b). C'est une évolution que les gouvernements des PVD doivent intégrer pour revendiquer de nouvelles aides à l'intensification auprès des agences bilatérales et multilatérales.

\section{Conclusion}

92 L'expérience chinoise dans l'utilisation de variétés génétiquement modifiées de coton est une réussite, du moins dans le court terme. Les variétés de CGM se révèlent efficaces, avec une efficacité qui s'est maintenue sept ans après leur commercialisation en 1998. Elles sont rentables pour les paysans qui les ont adoptées de manière quasi exclusive aujourd'hui dans la province du Hebei. Les paysans chinois bénéficient d'un très grand choix de variétés, ces variétés sont de surcroît en renouvellement permanent par un grand nombre d'acteurs relevant d'institutions chinoises de recherche de divers niveaux administratifs. C'est une situation très éloignée du monopole des firmes responsables de l'introduction du CGM. Globalement, l'expérience chinoise est donc une infirmation de la vision pessimiste relative à l'utilisation des variétés génétiquement modifiées par les petits paysans ou par les PVD.

La diffusion du CGM a bénéficié d'une combinaison d'un grand nombre de facteurs favorables dans les domaines technique, économique et institutionnel. Les facteurs techniques ont certes leur importance, ils s'appliquent à la technologie du CGM mais aussi aux technologies plus conventionnelles de création variétale et de mise au point de techniques culturales appropriées. Ces facteurs techniques ont découlé d'une recherche chinoise décentralisée qui a fonctionné dans la durée, par le seul soutien de l'État. Ils se sont exprimés également au niveau des paysans par une politique de soutien de l'État à l'intensification. 

du CGM. Le niveau d'intensification sur lequel l'utilisation du CGM a pu se greffer est fondamentalement le produit d'un puissant facteur institutionnel représenté par la volonté d'implication de l'État. C'est la poursuite de cette implication qui a permis de parvenir à des règles spécifiques et plus favorables de commercialisation du CGM que la Chine a réussi à faire valoir, même face à des firmes multinationales qui ont imposé des règles bien différentes dans d'autres pays. Nous soulignons ainsi l'importance des facteurs institutionnels qui est insuffisamment mise en avant dans les travaux antérieurs rapportant l'expérience chinoise : c'est un résultat qui confirme aussi la pertinence à considérer ensemble innovation technique et innovation institutionnelle (Fok, 2002a). rencontré dans d'autres pays, qu'ils relèvent de PVD ou de pays émergents. Il nous paraît donc abusif de considérer que les résultats de l'expérience chinoise peuvent être extrapolés à d'autres pays dans le monde. Même en Chine, la seule diffusion du CGM ne suffit pas pour garantir une production stable à haut niveau. Un changement d'ordre institutionnel, par un revirement dans le soutien, même discret, au prix d'achat du coton peut modifier fortement la rentabilité de la production cotonnière. C'est ce qui est observé actuellement dans la province du Hebei, avec une conversion substantielle des soles cotonnières en blé. dépend beaucoup des facteurs institutionnels. Il serait donc erroné de considérer qu'un changement technique, comme l'adoption de variétés génétiquement modifiées, suffirait à faire évoluer une production dans le sens désiré et de manière durable. C'est pourtant l'idée qui nous semble prédominer dans les prises de position en faveur de l'utilisation des variétés génétiquement modifiées et conduisant au concept de "Gene revolution ». Notre position rejoint l'analyse critique de Wu et al. (2004a) qui contestent l'assimilation de la disponibilité de variétés génétiquement modifiées à la révolution Verte en raison de la complexité des biotechnologies mises en œuvre, de l'implication essentielle du secteur privé et d'un climat géopolitique apaisé qui n'incite pas à aider les PVD à s'approprier du nouveau progrès technique. Le cas de la Chine nous pousse plutôt à considérer que la révolution des gènes ne serait qu'une étape plus poussée de la révolution Verte.

contribution se distingue aussi en identifiant plus précisément les facteurs favorables dans le cas du coton, en soulignant la nécessité d'une innovation institutionnelle d'accompagnement mais qui doit dépasser la seule reconnaissance de la propriété intellectuelle des variétés. Pour que d'autres PVD puissent tirer profit des produits des biotechnologies en agriculture, il faudrait relancer un véritable partenariat public/privé avec implication d'acteurs internationaux et réhabiliter le rôle de l'aide à l'intensification.

98 Enfin, le succès chinois reste une réussite de court terme, il n'est pas garanti de perdurer en raison de la menace d'un changement notable du complexe parasitaire, en direction de ravageurs plus difficiles à combattre. Dans ce domaine de menace à moyen terme, l'évolution en Chine indique que le danger ne viendra pas seulement de la résistance des ravageurs-cibles au CGM, crainte fréquemment évoquée pour ne pas dire exclusivement considérée dans les stratégies d'accompagnement d'utilisation du CGM. Le danger peut venir plus rapidement du franchissement du seuil de nuisibilité des ravageurs auparavant secondaires. Plus inquiétant est de constater que cette évolution ne provient pas forcément des ravageurs à incidence différente de celles des chenilles (comme les insectes 
piqueurs-suceurs), mais d'une autre chenille plus vorace et à croissance plus rapide. Comme dit un proverbe chinois, «il faut se garder de se flatter d'avoir chassé le loup de devant la porte sans se rendre compte du tigre qui est entré par-derrière... »

\section{BIBLIOGRAPHIE}

Bacheler J.-S., Mott, D.-W., Morrison D.-E. Large scale evaluation of bollgard resistance to multiple pests in North Carolina under grower conditions. Communication presented to Conference 'Beltwide Conference', 1998, 961-964 p.

Benhamou J.-F., Macrae J., Raymond G. Evaluation macro-économique de la filière coton et des effets du Contrat Plan sur la période 1989/90-1993/94 : Rapport de synthèse. CMDT, Bamao (Mali), mai 1992, 59 p.

Bennett R., Ismaël Y., Kambhampati U., Morse S. Economic impact of genetically modified cotton in India AgBioForum, 2004, 7, 3, p. 96-100.

Brooks N. Characteristics and production costs of US cotton farms. USDA, Washington, october 2001, $38 \mathrm{p}$.

Cabanilla L., Abdoulaye S.-T., Sanders H.-J. Economic cost of non-adoption of Bt Cotton in West Africa: with special reference to Mali. Communication presented to Conference "7th ICABR International Conference on « Public Goods and Public Policy for Agricutural Biotechnology »", Ravello (Italy), June 29-July 3, 2003, 20 p.

Carlson G.-A., Marra M.-C. Yield, insecticide use and profit changes from adoption of Bt cotton in the Southeast. Communication presented to Conference "Beltwide Cotton conferences", 1998, 973-974 p.

Chervel M., Fabre P., Kane R., Saldarriaga G. Manuel d'évaluation des projets d'investissement par la méthode des effets, Orbiter, 1997, $356 \mathrm{p}$

Edens E.-R., Slinsky, Larson J.-A., Roberts R.-K., Lentz G.-L. Economic analysis of genetically engineered Bt cotton for tobacco budworm and bollworm control. Communication presented to Conference "Beltwide Cotton Conferences", 1998, 380-383 p.

Elena M.-G. Economic advantage of transgenic cotton in Argentina. Communication presented to Conference "Beltwide Cotton Conferences", 2001, 1066-1068 p.

Fabre P. Manual Financial and economic analysis. European Union, Bruxelles. 1997, 375 p.

Falck-Zepeda J., Traxier G., Nelson R.-G. Rent creation and distribution from biotechnology innovations: the case of Bt cotton and herbicide-tolerant soybeans in 1997 Agribusiness, 2000, 16, 1, p. 21-32.

Falck-Zepeda J.-B., Traxler G., Nelson R.-G. Rent creation and distribution from the first three years of planting Bt cotton. ISAAA, New York, 1999, $18 \mathrm{p}$.

FAO. La situation mondiale de l'alimentation et de l'agriculture 2003-2004. Les biotechnologies agricoles : une réponse aux besoins des plus démunis? FAO, Rome, 2004, 227 p. 
Fitt G.-P. Utilisation et impact des cotons Bt transgéniques en Australie. ICAC recorder, Issue n

- décembre 2003, p. 16-22.

Fitt G.-P., Wilson L., Mensah R., Daly J. Advances with integrated pest management as a component of sustainable agriculture: the case of the Australian cotton industry. Communication presented to Conference "4th International Crop Science Congress", Brisbane, Australia, 2004, 11 p.

Fok A.-C.-M. État, production et exportation cotonnières, industrie textile et développement économique. Une histoire économique du coton/Textile dans le monde. Doctorat en Economie, Univ. Montpellier I, Montpellier, 1997, France. 827 p.

Fok A.C.M. (2002a). Cotton future in Western and Central Africa: the challenge of combining technical and institutional innovations. OCL, 2002, 9, 2-3, p. 115-122.

Fok A.C.M. (2002b). Intégration de l'agriculture dans les négociations internationales de l'OMC: comment saisir les opportunités offertes pour les filières cotonnières. Publié par CMA-AOC, 2002, site www.cmaoc.org

Fok A.C.M., Djouara H., Tomas C. Progress and challenges in making productivity gains cotton production by smallholders in Sub-Saharan Africa (SSA). Communication presented to Conference “World Cotton Research Conference 3", Cape Town, South Africa, 2004, 1515-1530 p.

Fok A.C.M., Koné M., Djouara H., Ballo D. Diversité des pratiques paysannes en zones cotonnières du Mali : portée et limites des gestions d'itinéraires techniques observées. Communication presented to Conference 'Rôle et place de la recherche pour le développement des filières cotonnières en évolution en Afrique’, Montpellier, 1-2 septembre 1999, 1999, 137-159 p.

Fok A.C.M., Liang W., Wang G., Wu Y.

I risultati positivi della diffusione del cotone Bt in Cina: limiti al trasferimento dell'esperienza cinese in altri paesi in via di sviluppo (Positive outcomes of the Bt cotton diffusion in China: limited scope of extrapolation to developing countries). Nuovo Diritto Agrario, 2005, 3/2004, p. 45-67.

Gouse M., Pray C.-E., Schimmelpfennig D.-E. The distribution of benefits from Bt Cotton adoption in South Africa. AgBioForum 2004, 7, 4, p. 187-194.

Hoag H. Biotech firms join charities in drive to help Africa's farms. Nature, 2003, 422, 246, p. 1.

Huang J., Hu R., Pray C.-E., Qiao F., Rozelle S. (2003a). Biotechnology as an alternative to chemical pesticides: a case study of Bt cotton in China. Agricultural Economics 29, 2003, p. 55-67.

Huang J., Hu R., Rozelle S., Qiao F., Pray C.-E. Transgenic varieties and productivity of smallholder cotton farmers in China. The Australian Journal of Agricultural and Resource Economics, 2002, 46, 3, p. 367-387.

Huang J., Hu R., van Meijl H., van Tongeren F. (2003b). Economic impacts of genetically modified crops in China. Communication presented to Conference "25th International Conference of Agricultural Economists", Durban, South Africa, 2003, 1075-1083 p.

Huang J., Pray C. E., Rozelle S. (2003c). Bt cotton benefits, costs and impacts in China. AgBioForum, 2003, 5, 4, p. 153-166.

ICAC. Survey of the cost of production of raw cotton. ICAC, Washington D.C., USA, september 2001, $112 \mathrm{p}$.

ICAC. Executive summary of the Report of the Second Expert Panel on Biotechnology of Cotton. ICA Recorder, 2004, XXII, 4, p. 4-8. 
Ismaël Y., Bennett R., Morse S. Benefits from Bt Cotton use by smallholders farmers in South Africa. AgBioForum, 2002, 5, 11, p. 1-5.

Kerby T.-A. What changes in yield and quality are due to transgenic varieties? The cotton gin and oil mill press, 2001, p. 5-7.

Kirsten J., Gouse M., Beyers L. Bt cotton in South Africa: adoption and the impact on farm incomes amongst small-scale and large scale farmers. University of Pretoria, Pretoria, 2002, 10 p.

Kowalski S.-P., Ebora R.-V., Kryder R.-D., Potter R.-H. Transgenic crops, biotechnology and ownership rights : what scientists need to know. The Plant Journal, 2002, 31, 4, p. 407-421.

Lague D. Biotechnology: the promise of food security. Far Eastern Economic Review, Hong Kong, 4 April 2002, p 6.

Li F. Prodenia litura (Fabricius): factors of its outbreak and how to control it. Anhui Agriculture, 2004, 7, p. 20.

Liang W., Fok A.C.M., Wang G., Wu Y. À part-time and female-managed agriculture: an orientation not sufficient to catch up the income gap in China. Communication presented to Conference "Colloque SFER « Les mutations de la famille agricole, conséquences pour les politiques publiques »", 22-23 Avril 2004, Paris, 16 p.

Magaña J.E.M., Garcia J.-G., Rodriguez A.-J. O., Garcia J. M. O. Comparative analysis of producing transgenic cotton varieties versus non-transgenic variety in Delicias, Chihuahua, Mexico. Communication presented to Conference "Beltwide Cotton Conferences", 1999, 255-256 p.

Marra M.-C., Pardey P.-G., Alston J.-M. The payoffs to transgenic field crops: an assessment of evidence. AgBioForum 2003, 5, 2, p. 43-50.

McGloughlin M. Ten reasons why biotechnology will be important to the developing world. AgBioForum, 1999, 2, 3, 4, p. 10.

Michotte F. Le coton, sa disparition prochaine et irrémédiable. L'erreur de sa culture. Société de propagande coloniale, Paris. 1924, $52 \mathrm{p}$.

Nyberg A., Chobanian E., Burcroff R.-I., Gilham F., Debatisse M., Zhu Z., Lacroix R., Bell T., SaintPierre C., Tuan F., Fry J., Chavancy G., Candiani G. China Agro-Industrial Crop Marketing. World Bank, Washington, June 17 1994, 162 p.

Orphal J. Comparative analysis of the economics of Bt and Non-Bt cotton production. Institute of economics in Horticulture, Univ. of Hannover, Hannover, Germany, January 2005, 71 p.

Pingali P., Raney T. Globalization and Agricultural Biotechnology Research: implications for the developing countries. Communication presented to Conference '85th Seminar, European Association of Agricultural Economists', Florence, Italy, Sept. 8-11 2004, 20 p.

Prakash C.-S. Global Hunger - Can Agricultural Biotechnology Help? Communication presented to Conference 'Feeding a Hungry World: The Moral Imperative of Biotechnology', Rome, Italy, 2004, $7 \mathrm{p}$.

Pray C.-E., Huang J., Hu R., Rozelle S. Five years of Bt cotton in China - the benefits continue. The Plant Journal, 2002, 31, 4, p. 423-430.

Pray C.-E., Ma D., Huang J., Qiao F. Impact of Bt cotton in China. World Development, 2001, 29, 5 , p. 813-825.

Qaim M., de Janvry A. Genetically modified crops, corporate pricing strategies, and Farmers' adoption: the case of Bt cotton in Argentina. American Journal Agricultural Economics, 2003, 85, 4, p. 814-828. 
Shelton A.-M. The role of plant biotechnology in the world's food system Economic Perspectives, 2003, 8, 3, p. 23-25.

Sheng C. Cotton pest control in Xinjiang's future. Cotton International, Issue No. issue, 2000, p. 107-108.

Taxler G., Falck-Zepeda J.-B. The distribution of benefits from the introduction of Transgenic cotton varieties. AgBioForum, 1999, 2, 2, p. 4.

Taylor M.-R., Cayford J. American Patent Policy, Biotechnology, and African Agriculture. The case for Policy change. Resource for the Future, Washington, november 2003, 112 p.

Teng K., Huang J. The commercial environment and product development for agricultural biotechnology in China. Centre for Chinese Agricultural Policy, Chinese Academy of Science, Beijing, 2004, 31 p.

Thirtle C., Jenkins Beyers L. Can GM-technologies help African smallholders? The impact of Bt cotton in the Makhatini Flats of Kwazulu-Natal World development, 2003, 31, 4, p. 717-732.

Traxler G., Godoy-Avila S. Transgenic cotton in Mexico. AgBioForum, 2004, 7, 1, 2, p. 57-62.

Wu F., Butz W.-P. (2004a). The future of genetically modified crops. Lessons from the Green Revolution. Rand Science and Technology, Santa Monica (CA, USA), 2004, 116 p.

Wu K. À brief statement on the studies of the ecological impact of Bt cotton conducted by Dr. Kongming Wu's lab. Institute of plant Protection, CAAS, 2002.

Wu K., Feng H., Guo Y. (2004b). Evaluation of maize as a refuge for management of resistance to Bt cotton by Helicoverpa armigera (Hübner) in the Yellow River cotton-farming region of China. Crop Protection, 2004, 23, p. 523-530.

Xu J., You Z., Wang W., Yang Y. Economic analysis of Bt cotton Planting in Jiangsu. Journal of Yangzhou University Agricultural and Life Science Edition, 2004, 25, 3, p. 65-69.

Zhang T., Zhou S. The economic and social impact of GMOs in China. China Perspectives, 2003, 47, p. 50-57.

\section{NOTES}

1. Mentionné H. armigera dans la suite de l'article.

2. . La fibre est le principal produit économique de culture. Même en considérant un cours mondial très bas, le kilogramme de fibre vaut 1 \$ US, contre 0,10 \$ US pour la graine, en position FOB. Dans beaucoup de pays, les fermiers abandonnent la propriété de leurs graines de coton pour payer le service d'égrenage.

3. . À raison de 300 unités d'azote, de 105 unités de P205 et de 125 unités de K2O dans la province du Jiangsu.

4. . Jusqu'au milieu des années 1990 , l'industrie textile était la première source de devises de la Chine. Ce n'est plus le cas aujourd'hui et entre-temps, la Chine est la deuxième réserve de devises dans le monde depuis fin 2004.

5. . Ce constat s'oppose à l'idée véhiculée par certains organismes internationaux spécialisés dans le coton, idée reprise dans la protestation des pays africains à Cancun en septembre 2003. Cette idée est discutable et son approfondissement, que nous avons abordé (Fok, 2005), nous éloignerait du sujet.

6. . Au cours de la période 1980-2003, on recense la vulgarisation effective de 176 variétés différentes de coton. 
7. . Il semble bien que les chercheurs ne peuvent plus introgresser des gènes sans le respect du droit de propriété, par contre il est difficile pour un étranger de percer le statut des variétés ayant été introgressées antérieurement.

8. . Théoriquement, un paysan n'a pas intérêt à resemer les graines issues de sa parcelle semée avec une variété hybride, c'est ce que nous voulons étudier dans les travaux que nous venons de démarrer dans cette vallée (différents de ceux que nous rapportons ici) et qui indiquent déjà que la diffusion du CGM semble avoir profité de la présence de deux innovations techniques complémentaires.

9. . À notre connaissance, il n'y a pas d'étude disponible pour situer l'importance relative de cette habitude.

10. . Pour les adversaires du coton-Bt, cette synthèse permanente de toxine Bt devrait avoir pour effet d'accentuer la sélection de chenilles résistantes.

11. Le marché des semences de CGM semble avoir les traits d'un " marché contestable ", avec de très faibles coûts à l'entrée et à la sortie, mais cela est un autre sujet.

12. . En raison de la pratique d'utilisation des semences fermières produites par le paysan et des échanges entre les paysans, il n'est pas facile d'identifier les variétés utilisées.

13. . L'indice A de Cotlook de Liverpool sert de référence de prix mondial. Cet indice est estimé en position de vente CAF en Europe du Nord. Pour ramener cet indice à la position bord champ, nous avons retenu 9 cents US/livre. Nous avons décompté aussi 3 cents US/livre pour l'égrenage afin de séparer le coton fibre du coton-graine (avec un rendement moyen de $36 \%$ ).

14. . Nous observons une répercussion plus directe du prix mondial du coton pour la campagne 2003/2004. Le gouvernement chinois a décidé de relancer les productions de céréales, en allouant en particulier pour la première fois un paiement direct aux paysans. La répercussion de la baisse du prix mondial est à l'origine de la conversion des soles cotonnières en blé pour la campagne $2004 / 2005$.

\section{RÉSUMÉS}

Les résultats d'une enquête récente en Chine confirment le succès de l'utilisation de variétés génétiquement modifiées de coton. Cette réussite n'est pas uniquement due aux qualités intrinsèques de ces variétés. Elle provient d'un ensemble favorable de facteurs techniques et économiques et surtout à des règles de diffusion très différentes de celles imposées dans les autres pays.

Successful diffusion of genetically modified varieties of cotton in China is being confirmed by the outputs of a recent survey. This success does not derive only from the intrinsic advantages of these varieties but from a set of favourable technical and economical factors, furthermore from specific diffusion rules very different from those imposed so far elsewhere in the world.. 
INDEX

Keywords : china, cotton, GMO, institution, seed marketing

Mots-clés : chine, commercialisation des semences, coton, institution, OGM

Code JEL Q16 - R\&D; Agricultural Technology; Agricultural Extension Services, Q15 - Land

Ownership and Tenure; Land Reform; Land Use; Irrigation, Q18 - Agricultural Policy; Food Policy

\section{AUTEURS}

\section{MICHEL FOK AH CHUEN}

CIRAD et UMR Moïsa, Montpellier, France

\section{YUHONG WU}

Hebei Agricultural University, Baoding, République populaire de Chine 